\title{
Le juge est (souvent) une femme Conceptions du métier et pratiques des magistrates et magistrats aux Affaires familiales
}

\author{
The Judge is (Often) A Woman \\ Professional Perceptions and Practices of Male and Female Family Court Magistrates
} Céline Bessière*, a et Muriel Mille ${ }^{b}$

${ }^{a}$ Université Paris-Dauphine/Institut de recherche interdisciplinaire en sciences sociales (IRISSO), Place du $\mathrm{M}^{1}$ de Lattre de Tassigny, 75775 Paris cedex 16, France

${ }^{a}$ Centre Maurice Halbwachs, 48 boulevard Jourdan, 75014 Paris, France

b Institut Marcel Mauss, 190-198 avenue de France, 75013 Paris, France

Auteur correspondant : Céline Bessière

Adresses e-mail : celine.bessiere@,dauphine.fr (C. Bessière); millemuriel@gmail.com (M. Mille)

\begin{abstract}
Résumé
En France, le contentieux des séparations conjugales est traité dans les Chambres de la famille des tribunaux de Grande instance (TGI) par un juge unique (un ou une juge aux Affaires familiales). Dans un contexte de féminisation de la magistrature, la question de l'influence du sexe du juge sur les décisions prises aux Affaires familiales est devenu un sujet sensible, sous la pression des associations de défense des droits des pères qui en ont fait un cheval de bataille médiatique. En s'appuyant sur les résultats d'une enquête collective menée dans quatre TGI entre 2008 et 2010, l'article montre que les juges aux Affaires familiales hommes et femmes n'ont pas la même trajectoire personnelle et professionnelle, qu'ils n'arrivent pas à cette fonction dans les mêmes conditions, au même moment de leur carrière et par là même qu'ils n'ont pas la même manière d'envisager leur rôle. Malgré toutes ces différences, il règne une grande homogénéité des décisions, quel que soit le sexe du juge.
\end{abstract}

Mots clés: genre; justice; trajectoires professionnelles; magistrature ; séparations conjugales ; féminisation.

\begin{abstract}
In France, disputes related to marital separations are heard in the Family Chambers of the Superior Courts (Chambres de la famille des Tribunaux de Grande instance) by a single judge (a family court judge). As the magistracy feminizes, the question of what influence a judge's gender has over rulings in family cases has become a controversial topic and a media hobbyhorse, under mounting pressure from organizations that defend fathers' rights. Using the results of a collective survey conducted in four Superior Courts between 2008 and 2010, this article shows that male and female family court judges do not follow the same personal and professional paths and do not assume their jobs under the same conditions or at the same moment in their careers, and thus perceive their roles in quite different ways. Despite all these differences, there is great homogeneity in these judges' decisions, no matter their sex.
\end{abstract}

Keywords : Gender; justice; career paths; magistracy; marital separations; feminization.

Depuis le début des années 2000, les femmes représentent plus de la moitié des juges en poste (58\% en 2011) alors qu'elles n'ont accédé à cette fonction que depuis 1946 et qu'elles représentaient seulement $6 \%$ des magistrats en 1959 (Boigeol, 1993). En 2011, trois quarts 
des juges qui sortent de l'École nationale de la magistrature (ENM) sont des femmes.

Dans les Chambres des Affaires familiales, où est traité le contentieux massif des séparations conjugales (divorces, litiges entre parents non mariés ou post-divorces), cette féminisation de la magistrature est devenue un sujet sensible. En effet, les Affaires familiales opposent presque toujours un justiciable homme à une justiciable femme ${ }^{1}$, sur des litiges ayant trait à la division du travail parental et à l'économie domestique, soient des questions éminemment genrées. Ces contentieux sont traités par un juge unique, le "juge aux Affaires familiales », ou bien plus souvent aujourd'hui — dans environ 7 cas sur 10 - par une « juge aux Affaires familiales $\gg(\mathrm{JAF})^{2}$.

La question de l'influence du sexe du juge sur les décisions prises en matière de résidence des enfants ou de pension alimentaire est devenue un cheval de bataille médiatique pour des associations de défense des droits des pères qui dénoncent une justice rendue par des femmes ${ }^{3}$ pour des femmes. «Les femmes jugent avec leurs tripes [...]. L'hyper-féminisation des professions de la magistrature pose un vrai problème! Les femmes comprennent les femmes [...]. Tout comme les femmes qui souhaitent être représentées par leur genre au sein de la politique, les papas que nous sommes demandons la même chose dans la magistrature. Un juge devrait être asexué, car il décide de la vie des personnes » déclarait récemment Fabrice Mejias, président de l'association SOS Papa ${ }^{4}$.

Cette argumentation sexiste n'est pas nouvelle. Anne Boigeol a montré combien la féminisation de la magistrature a suscité des résistances très fortes au sein de la profession. Dans les années 1950, les rapports des jurys de concours de l'ENM rédigés par de hauts magistrats s'interrogeaient sur l'aptitude des femmes à exercer la profession (qualité du timbre de la voix, capacité d'autorité, de raisonnement et de maîtrise de soi) ; et dans les années 1970, tandis que la féminisation de la magistrature était désormais inéluctable, les jurys ont commencé à s'inquiéter du « péril » que représentait la féminisation « excessive » de la profession (Boigeol, 1996, pp. 116-121). Durant toute leur histoire, les femmes professionnelles de la Justice, plus souvent que les hommes, ont été soupçonnées d'un manque d'impartialité dans l'exercice de leur fonction (Cacouault-Bitaud, 2001) : les propos du président de SOS Papa réactivent ce schème sexiste avec virulence, les hommes incarnant la Justice neutre asexuée, tandis que les femmes sont renvoyées à leur nature (Rennes, 2007). Aujourd'hui, dans un contexte de massification du contentieux des Affaires familiales, la

${ }^{1}$ Les couples homosexuels apparaissent très rarement dans les procédures judiciaires de séparation, du fait de l'interdiction jusqu'à présent du mariage homosexuel en France mais aussi du fait de la difficile reconnaissance des parents de même sexe par le droit. Ce dernier point est en cours d'évolution. Depuis 2007, plusieurs Chambres des Affaires familiales en France (TGI de Lille, Créteil, Aix-en-Provence, Bayonne) ont attribué une délégation de l'autorité parentale au conjoint ou ex-conjoint de même sexe du parent, souvent au nom des « circonstances particulières mentionnées par la loi » (article 377-1). Plusieurs de ces décisions ont été cassées en Cour d'appel, à la demande du Parquet.

${ }^{2}$ La loi n $93-22$ du 8 janvier 1993 a supprimé le juge aux Affaires matrimoniales, créé en 1975 et exerçant en tribunal d'instance, au profit du JAF, exerçant en TGI. En 2011, selon le ministère de la Justice, les femmes représentent $68 \%$ des juges non spécialisés des TGI tous grades confondus, ce qui constitue la quatrième fonction la plus féminisée, derrière juge des enfants, juge d'instance, juge d'application des peines. Ce n'est qu'une fois affectés dans un TGI que les magistrates et magistrats sont nommés par le président du tribunal aux Affaires familiales ou à d'autres fonctions du TGI (en tant que juge du siège civil ou pénal). Le ministère de la Justice ne comptabilise pas le nombre d'hommes et de femmes JAF au niveau national.

${ }^{3}$ La féminisation des professions de la Justice ne concerne pas seulement les juges. Récemment, le nombre d'avocates vient de surpasser celui des avocats : elles représentent $51 \%$ des avocats tous barreaux confondus en 2010 (Moreau, 2010). L'histoire de la féminisation du greffe est plus ancienne : elle s'inscrit dans la féminisation des bureaux au début du $\mathrm{XX}^{\mathrm{e}}$ siècle et s'est accentuée avec la fonctionnarisation des greffiers depuis les années 1960 (Bossis 2003). En 2009, 87 \% des greffiers sont des femmes.

${ }^{4}$ Gay, C. 2012. Justice : entre femmes juges et prévenues, existe-t-il une solidarité féminine ? Le Point, 31 août. 
pression des groupes de pères ravive les résistances du corps de la magistrature à sa féminisation. La question du manque d'hommes et de la présence en (trop) grand nombre de femmes dans les Chambres de la famille est devenu un "problème " à résoudre pour l'institution judiciaire ${ }^{5}$.

Alors que dans les pays de common law, notamment aux États-Unis, des universitaires et des juristes se sont intéressés dès les années 1970 aux effets du sexe du juge sur les décisions judiciaires rendues ${ }^{6}$, peu de travaux existent dans les pays de droit civil ${ }^{7}$, et encore moins en France $^{8}$ (Boigeol, 2010). Le courant de recherches gender and judging, florissant depuis les années 1990, nous apprend qu'il ne suffit pas de s'intéresser quantitativement aux différences de décisions prises par les juges hommes et femmes, mais qu'il faut considérer tout le processus qualitatif du jugement - la manière et les raisons avec et pour lesquelles les juges jugent (Hunter, 2013). Dans le prolongement de ces travaux, nous analyserons des trajectoires d'hommes et de femmes juges aux Affaires familiales pour saisir les éventuelles différences dans leurs pratiques et leur conception du métier.

Les résultats présentés dans cet article reposent sur une enquête collective qui a réuni une trentaine de sociologues (enseignants-chercheurs, doctorants et étudiants en Master) et qui a donné lieu à un rapport pour la mission de recherche Droit et Justice (Bessière et al., 2010). Cette enquête est menée depuis 2008 dans les Chambres de la famille de quatre TGI en France dont les formes organisationnelles (de 3 à $12 \mathrm{JAF}$ ) et les bassins d'emploi correspondant à leur juridiction varient (deux tribunaux en région parisienne, deux en province, dont un dans une grande ville, l'autre dans un département plutôt rural) ${ }^{9}$. Dans notre enquête, sur $28 \mathrm{JAF}$ en poste, il y avait 20 femmes et 8 hommes. Après avoir présenté brièvement les conditions de travail des juges aux Affaires familiales, nous allons montrer que les magistrates et les magistrats qui occupent cette fonction n'y arrivent pas pour les mêmes raisons, ni au même moment de leur carrière. Par conséquent, ils n'ont pas la même manière d'envisager leur rôle. Pour finir, nous examinerons l'influence du sexe du juge sur les décisions prises aux Affaires familiales.

\section{Encadré 1 : Méthodologie de l'enquête}

L'observation des audiences à différents moments et dans le cadre de différents types de procédure de séparation conjugale (consentement mutuel, audiences de non-conciliation, litiges post-divorces et contentieux de couples non mariés) a constitué le cœur de l'enquête ethnographique. Trente-neuf audiences d'une demi-journée menées par 16 juges différents ont

\footnotetext{
${ }^{5}$ En juin 2012, l'ENM organisait une journée de formation continue sur le thème de la féminisation à laquelle nous avons été conviées pour présenter notre recherche. Les intitulés des sessions attestent de la manière dont l'institution judiciaire constitue la féminisation comme " problème » : « Pourquoi tant de femmes à l'ENM ? ; «Les femmes rendent-elles la Justice autrement?». Le 17 décembre 2012, la Garde des Sceaux en visite à l'ENM de Bordeaux s'est également prononcée — prudemment — sur ce thème : " progressivement, nous aurons à faire en sorte qu'il y ait plus d'hommes dans les prochaines promotions, mais je pense que les garçons vont revenir assez vite» (cf. Magistrature trop féminisée, Christiane Taubira veut attirer plus d'hommes. LexTimes.fr, 17 décembre 2012).

${ }^{6}$ Ces travaux s'inscrivent, encore aujourd'hui, dans un tout autre contexte institutionnel où les femmes juges sont nettement minoritaires dans la magistrature. Pour une revue de littérature récente, voir Feenan (2009).

${ }^{7}$ On peut néanmoins souligner la présence de travaux récents sur l'influence du sexe du juge sur le processus de prise de décision judiciaire en matière familiale, au Brésil (Bothelho Junqueira, 2003) et en Argentine (Kohen 2008).

${ }^{8}$ Les recherches françaises sur la justice introduisant une perspective de genre dans l'analyse ont surtout porté sur les caractéristiques des justiciables. Dans les affaires pénales, ces travaux montrent que les sanctions judiciaires sont plus clémentes à l'égard des femmes que des hommes (F.-L. Mary, 1996; Lelièvre et Léonard, 2012).

${ }^{9}$ Par souci d'anonymisation, les quatre TGI seront ici désignés par des noms de juridiction fictifs (Belles, Carly, Marjac et Valin). Tous les noms des professionnels de la Justice ont également été modifiés.
} 
été observées en binôme, soient 330 affaires. L'observation a été complétée par la consultation d'une centaine de dossiers judiciaires correspondant à certaines des affaires suivies en audience, de manière à mieux situer cette phase orale au sein de la procédure écrite. Elle s'est aussi accompagnée d'entretiens enregistrés et non enregistrés avec les JAF $(\mathrm{N}=21)$. Parce que notre accès au terrain s'est fait par le biais des Chambres de la famille des TGI, et que nous avons fréquemment été assimilés à des professionnels de la Justice par les parties présentes aux audiences, nous avons fait le choix de ne pas faire d'entretien avec les justiciables.

Le volet quantitatif de l'enquête est fondé sur le dépouillement et le codage de dossiers judiciaires $(\mathrm{N}=400)$. Nous avons traité un échantillon aléatoire de 100 dossiers archivés entre le 15 mai 2007 et le 15 juin 2007, puis entre le 15 octobre et le 15 novembre 2007, dans chacun des TGI où nous avons mené notre enquête ethnographique. La saisie des dossiers (en trois bases distinctes, selon le type de procédure : consentement mutuel, divorces contentieux, hors et post-divorce) a respecté le principe de confidentialité des sources; elle s'est efforcée de conserver la richesse de la dimension diachronique des dossiers et de rendre compte de la pluralité des points de vue exprimés.

\section{Des juges face à un contentieux de masse}

En France, le principe de confier aux JAF l'ensemble des divorces, y compris ceux par consentement mutuel, a été récemment réaffirmé ${ }^{10}$, tandis que la médiation familiale est peu développée (Bastard, 2010). En somme, plutôt que réduire le nombre de dossiers confiés aux juges, notre pays a choisi de simplifier les procédures, en désignant un juge unique (plutôt qu'une formation collégiale) dans la quasi-totalité des procédures et surtout en encourageant le consentement mutuel, synonyme de procédure accélérée. Ainsi, les Affaires familiales représentent environ la moitié du contentieux civil dans les TGI ${ }^{11}$. En 2010, les 28 JAF en poste dans les quatre juridictions enquêtées ont rendu 21545 décisions, soit une moyenne de 769 décisions annuelles par juge.

Chaque semaine, les juges rencontrés tiennent trois à quatre audiences d'une demi-journée aux Affaires familiales. Pour chacune, 14 à 15 affaires sont prévues: certaines sont renvoyées, d'autres donnent lieu à une décision immédiate lorsque les conjoints parviennent à un accord à l'audience. Pour celles qui restent, les juges annoncent aux justiciables une date de délibéré (environ deux à trois semaines pour les audiences de conciliation et les affaires hors-mariage ; environ un à deux mois pour les jugement de divorce sur le fond), et rédigent dans ce délai leur décision, envoyée ensuite aux parties. Ce travail de rédaction occupe une grande partie du temps laissé libre par les audiences, même si les JAF tiennent aussi occasionnellement des audiences en correctionnelle, siègent aux assises ou remplacent des collègues absents dans d'autres fonctions de leur juridiction.

Dans un contexte d'une évaluation grandissante de l'activité judiciaire par des indicateurs quantitatifs, l'augmentation du contentieux des Affaires familiales et la durée de traitement

\footnotetext{
${ }^{10}$ Voir le rapport de la Commission sur la répartition des contentieux remis au Garde des Sceaux : Guinchard, S. (dir.), 2008. L'Ambition raisonnée d'une Justice apaisée. La Documentation française, Paris. Cette réaffirmation de la place des juges dans le contentieux familial fait suite à la recommandation du premier conseil de modernisation des politiques publiques de confier aux notaires l'homologation des divorces par consentement mutuel (12 décembre 2007). La déjudiciarisation d'une partie des divorces est ainsi souhaitée par les acteurs de la réforme de l'État (au service de la Révision générale des politiques publiques, RGPP) mais refusée jusqu'à présent par les professionnels de ce contentieux.

11 Selon les données nationales du ministère de la Justice, entre 2005 et 2011, les Affaires familiales ont représenté de $47 \%$ à $50 \%$ du stock des dossiers civils des TGI, de $44 \%$ à $46 \%$ des nouvelles affaires et de $41 \%$ à $46 \%$ des affaires terminées une année donnée (hors référé*).
} 
des affaires sont l'objet de préoccupations de la part des autorités gestionnaires, depuis la Chancellerie jusqu'aux présidents des Chambres de la famille (Vigour, 2011). L'inspiration managériale des réformes de la Justice donne une légitimité accrue à ces indicateurs, en rupture avec sa traditionnelle «exceptionnalité » qui par ses idéaux de singularité et d'incommensurabilité conduisait à valoriser la lenteur des procédures et à empêcher l'application des standards d'efficacité en vigueur dans d'autres services publics ${ }^{12}$.

Les JAF sont ainsi soumis à des contraintes managériales de productivité résumées dans « les statistiques » de leur cabinet et étudiées de près par leur hiérarchie, notamment, quant au nombre de décisions rendues, aux délais de jugement, à la réduction du stock de dossiers en cours. Peu maîtres du nombre d'affaires programmées chaque semaine, les JAF doivent « tenir la cadence», pour ne pas «crouler sous les dossiers ». Ainsi, le temps accordé à chaque couple est faible. Sur l'ensemble des 330 affaires que nous avons observées, la durée moyenne de chaque audience s'établit à 18 minutes (pour une amplitude de 3 minutes à 1h30). Les juges peuvent certes avoir recours à des mesures d'investigation (notamment des enquêtes sociales), voire à des auditions d'enfant, qui permettent de recueillir des informations complémentaires, mais ces mesures représentent un surcoût que les justiciables ou la puissance publique (en cas d'aide juridictionnelle) doivent assumer et, allongeant le temps nécessaire au rendu du jugement, elles sont donc utilisées avec parcimonie.

Les JAF se trouvent ainsi soumis à des injonctions contradictoires : être à l'écoute des justiciables, rendre des décisions solides pour éviter le recours contentieux, le tout en étant réactif et prompt face à une masse toujours plus importante de dossiers. Plusieurs JAF se sont montrés critiques à l'égard de cette « logique comptable» (selon l'expression de l'une d'entre eux), estimant qu'elle risquait de nuire à la «qualité » des décisions rendues. Nous allons montrer que confrontés à cette même mission impossible, les femmes et les hommes JAF, en pratique, la remplissent différemment.

\section{Des femmes juges des enfants reconverties aux Affaires familiales}

Concernant neuf des treize magistrates que nous avons interviewées, le passage préalable par la fonction de juge des enfants constitue la trajectoire modale des femmes JAF. Il s'articule avec un mode d'accès particulier à la magistrature (excellence scolaire et intérêt précoce pour la fonction) ainsi qu'à une conception interventionniste de leur rôle à l'égard des justiciables.

2.1 Juge des enfants par vocation

Anciennes (très) bonnes élèves, ces magistrates avaient souvent un objectif précis tout au long de leurs études de droit : devenir juge et particulièrement, juge des enfants. Cette « vocation » correspond à des réalités statistiques connues : la fonction de juge des enfants est de loin la plus féminisée de l'ensemble de la magistrature ${ }^{13}$. De surcroît, les carrières féminines dans la magistrature sont marquées par l'excellence scolaire (Boigeol 1993: 500). Ayant très bien réussi leurs études secondaires et supérieures, les femmes empruntent davantage que les hommes la « voie royale » pour l'accès au statut de magistrat, c'est-à-dire le premier concours d'entrée à l'ENM, qui s'adresse aux jeunes diplômés de niveau «bac +4 » et plus. En 2011, plus de $80 \%$ des reçus à ce premier concours étaient des femmes. Et à leur sortie de l'ENM, au moment où les élèves émettent des vœux d'affectation, les femmes choisissent plus souvent que les hommes un poste de juge des enfants ${ }^{14}$.

\footnotetext{
12 Ce basculement a d'abord été observé dans le contentieux pénal où, dès le milieu des années 1990, le traitement en temps réel a conduit à une accélération du rythme de la Justice (Bastard, Mouhanna, 2007 ; Christin, 2008). Il concerne aujourd'hui l'ensemble de l'activité judiciaire (Vauchez, 2008).

${ }^{13}$ Pour le ministère de la Justice en 2011, près de $80 \%$ des juges des enfants sont des femmes.

14 Dans les années 1990, $26 \%$ des auditrices souhaitaient occuper cette fonction à la sortie de l'ENM contre
} 
Aurélie Vermeer incarne bien ce type de trajectoire féminine. C'est une jeune magistrate âgée d'à peine 30 ans qui est JAF à Carly, un grand tribunal de la région parisienne, depuis septembre 2008. Fille d'un technicien dans une sucrerie et d'une employée EDF, elle a découvert la fonction de juge des enfants au collège, après la lecture d'un roman (Chiens perdus sans collier ${ }^{15}$ ). Dès la classe de $3^{\text {me }}$, âgée de 14 ans, elle avait organisé une visite du Palais de Justice en tant que déléguée de classe, assisté à des audiences et rencontré des magistrats. Aurélie Vermeer est entrée à l'ENM à 22 ans, directement après une maîtrise de droit, contrairement à $60 \%$ des étudiants de sa promotion qui y sont entrés plus âgés, avec un diplôme de troisième cycle. Elle a demandé comme premier poste sa " fonction préférée »juge des enfants - qu'elle a obtenue dans l'Est de la France et qu'elle a exercée « avec plaisir et parfois difficulté ». Au bout de deux ans, pour se rapprocher de la région parisienne dont elle est originaire, elle a demandé la fonction généraliste de juge de TGI. Elle a été affectée à Carly où le président du tribunal lui a proposé d'être nommée JAF ${ }^{16}$.

\subsection{De juge des enfants à JAF : le poids des contraintes familiales et professionnelles}

L'arrivée aux Affaires familiales de magistrates anciennement juges des enfants résulte d'un « choix » sous multiples contraintes. Des contraintes liées à l'organisation de la magistrature et des tribunaux d'une part. Pour avancer dans leur carrière, les juges sont en effet poussés à changer régulièrement de juridiction et de fonction. Ils émettent alors des vœux, en tenant compte de leurs ambitions professionnelles et de leurs impératifs familiaux. Une fois affectés à un TGI, les juges se voient proposer par le président du TGI les postes disponibles ; or, en raison de la masse du contentieux, il s'agit bien souvent de postes de JAF. De plus, les présidents des TGI — souvent des hommes ${ }^{17}$ — considèrent que les anciennes juges des enfants sont des candidates idéales au poste de JAF. Ainsi chaque mois de septembre, le président du tribunal de Carly reçoit la trentaine de magistrats qui lui sont affectés. Dans ses décisions, il tient compte des postes à pourvoir ainsi que des expériences précédentes et des vœux des juges, mais il se réserve aussi le droit de choisir des personnes qui ont le «profil » pour le poste : "JAF, c'est une fonction d'écoute, d'attention, de sensibilité ${ }^{18}$ ». Nombre de jeunes femmes s'étant, dans un premier temps, spécialisées dans la fonction de juge des enfants apparaissent aux présidents des TGI comme des recrues idéales pour les Affaires familiales, du fait de la proximité supposée entre les deux fonctions.

Par ailleurs, le passage de juge des enfants à JAF est souvent contraint par des raisons familiales qui s'imposent davantage aux juges femmes qu'aux juges hommes ${ }^{19}$ : il est frappant que dans les entretiens que nous avons réalisés avec les magistrates et les magistrats, ces derniers ont pu parfois passer sous silence leur situation familiale (présence d'un conjoint ou d'enfants), tandis que les femmes ont toujours été amenées à les mentionner pour raconter leur trajectoire professionnelle. Une partie des femmes juges ont par exemple suivi leur mari — souvent cadre dans le secteur privé (Boigeol, 1993: 492) — au gré des mutations professionnelles de celui-ci, ou changé de fonction à la naissance de leurs enfants ${ }^{20}$. Au

seulement $15 \%$ des auditeurs (Boigeol, $1993: 494$ ).

${ }^{15}$ Ce roman de Gilbert Cesbron paru en 1954 raconte le travail d'un juge des enfants face à des mineurs délinquants : c'est un succès de librairie, réédité de nombreuses fois

${ }^{16}$ Informations recueillies lors d'un entretien enregistré réalisé par Jérémy Mandin, le 9 février 2009.

${ }^{17}$ En 2011, 73 \% des chefs de juridiction sont des hommes.

${ }^{18}$ Entretien avec le président du TGI de Carly (homme, environ 55 ans), en présence de la secrétaire générale du TGI (femme, environ 45 ans), réalisé par Hélène Steinmetz et Jérémy Mandin, le 8 décembre 2008.

${ }^{19}$ On retrouve ici un résultat des travaux d'A. Boigeol sur les auditeurs et les auditrices de Justice à l'ENM : si la majorité des auditeurs déclare privilégier la fonction à la sortie de l'ENM, les auditrices sont plus nombreuses à privilégier un critère géographique, contrainte familiale oblige (Boigeol, 1993, p. 500).

${ }^{20}$ Comme l'écrit justement Armelle Testenoire : «alors que les hommes tendent à se percevoir et à se présenter 
moment de la mise en couple ou de la naissance de leurs enfants, la fonction de JAF leur apparaît comme une fonction moins "prenante ", moins " épuisante » que celle de juge des enfants, et donc davantage compatible avec leurs engagements familiaux.

Âgée d'une trentaine d'années, Sandrine Cabernet est JAF au TGI de Valin depuis 2 mois, une fonction qu'elle occupe à $80 \%$. C'est son deuxième poste, après une expérience comme juge des enfants dans une petite juridiction de la même région. Elle est mariée avec un cadre commercial qu'elle présente comme très accaparé par son travail (peu de vacances, ni de temps libre) et a deux enfants en bas âge. Habitant dans ce qu'elle nomme elle-même « un trou paumé »" à une trentaine de kilomètres de Valin, elle est très attentive au temps de transport entre son domicile et le tribunal et à la fréquence des trajets. Par rapport à la fonction de juge des enfants qui supposait une présence quotidienne au tribunal du fait des nombreuses permanences, elle apprécie la possibilité que lui permet le poste aux Affaires familiales de travailler plus souvent chez elle : « je ne viens vraiment pas tous les jours. Je rédige beaucoup chez moi [...] Donc là, c'est quand même plus cool [que juge des enfants], faut le reconnaître $»^{21}$. Par contraste avec leur fonction précédente et bien que la masse des dossiers à traiter soit conséquente, le poste de JAF fournit des conditions de travail que les jeunes femmes ayant charge de famille apprécient particulièrement, notamment la possibilité de réaliser l'essentiel du travail de rédaction à domicile, le soir ou le week-end. Alors que tous leurs collègues hommes travaillent à temps complet, certaines, comme la juge Sandrine Cabernet, exercent leur activité à temps partiel ${ }^{22}$.

Des juges femmes plus âgées, installées dans le poste de JAF parfois depuis plusieurs années, apprécient également les conditions de travail aux Affaires familiales, et continuent à penser la fonction de JAF en la rapportant à celle de juge des enfants. La juge Catherine Blanchard a une cinquantaine d'années ; vice-présidente, elle est chef du service des Affaires familiales du tribunal de Carly depuis 1996. Elle a occupé auparavant plusieurs fonctions de juge des enfants dans différentes juridictions et compare les deux métiers, en les liant explicitement aux transformations de sa vie familiale. Elle décrit ainsi les lourdes contraintes horaires de son précédent poste où il lui arrivait souvent de rester au tribunal jusqu'à $20 \mathrm{~h} 30$ :

"Juge des enfants, vous savez l'heure à laquelle vous partez le matin, vous ne savez pas
l'heure à laquelle vous rentrez le soir. Parce que si à $18 \mathrm{~h}$, il y a un travailleur social qui vous
envoie un signalement en vous disant: "dans la situation machin ça merde complètement, il
faut trouver une solution"» vous allez pas dire " bon écoutez, bye bye, il est $18 \mathrm{~h}$, c'est l'heure
de rentrer, je m'en vais !"23»».

Mariée à l'âge de 39 ans avec un ingénieur dans l'aéronautique, elle a eu un enfant en 1995 et est devenu JAF l'année suivante. Travaillant désormais moins « dans l'urgence et dans l'imprévu », elle considère ce poste comme «moins accaparant» et donc «plus facilement gérable » sur le plan personnel.

Les femmes juges qui sont entrées dans la magistrature pour devenir juge des enfants considèrent que le poste de JAF offre de meilleures conditions de travail, particulièrement appréciables lorsque leurs charges familiales s'alourdissent. Cependant, rares sont celles qui

comme autonomes face à leurs choix professionnels, les femmes se vivent comme membres de la communauté conjugale sur leur lieu de travail » (Testenoire, 2001, p. 133). Cette analyse produite au sujet de couples de doubles actifs de milieu populaire (ouvriers et employés) s'applique ici à des couples de professions intellectuelles et supérieures, preuve ici que les inégalités de genre traversent l'espace social.

${ }^{21}$ Entretien réalisé par Alina Surubaru et Elodie Hennequin, le $1^{\mathrm{er}}$ mars 2010.

${ }^{22}$ En avril 2012, sur 1676 juges non spécialisés dans les TGI, 95 ont un temps partiel à $80 \%$ et 35 un temps partiel de $50 \%$ à $70 \%$. Ce sont toutes, sans exception, des femmes. Rapporté au nombre de femmes juges non spécialisées dans les TGI $(\mathrm{N}=1134)$, on obtient $11,5 \%$ de femmes à temps partiel (Source : ministère de la Justice).

${ }^{23}$ Entretien réalisé par H. Steinmetz et J. Mandin, le 1er décembre 2008. 
comme C. Blanchard font des carrières très longues aux Affaires familiales. Certes, les juges du siège sont inamovibles, c'est-à-dire qu'ils ne peuvent recevoir, sans leur consentement, une affectation nouvelle. Mais le système d'avancement dans la magistrature pousse au changement régulier de fonction et de juridiction. Pour autant, les magistrates rencontrées assurent que la carrière n'est pas nécessairement un enjeu primordial pour elles ${ }^{24}$, même si certaines ambitionnent malgré tout d' «avancer». Aurélie Vermeer ironise : "Tout le monde ne veut pas être président de tribunal! ». D'autres expliquent qu'elles auront le temps de faire carrière en dehors des Affaires familiales quand leurs enfants auront grandi. Ainsi, bien que la magistrature soit largement féminisée, les ingrédients de la formation d'un «plafond de verre » en défaveur des femmes sont réunis du fait d'une gestion sexuée des compétences et des carrières ainsi que de l'inégale emprise du temps familial sur les hommes et les femmes juges (Laufer, 2004).

\subsection{Femmes JAF : posture pédagogique, recherche du consensus et intervention sociale}

Dans tous les cas, le passage par le tribunal des enfants s'est révélé marquant pour les femmes JAF : elles gardent une certaine nostalgie de leur première fonction qu'elles jugeaient plus « passionnante ». Rétrospectivement, elles appréciaient le suivi en profondeur des dossiers des mineurs sur un temps long, en coopération avec de nombreux intervenants : parents, éducateurs, assistantes sociales, pédopsychiatres, etc. On peut remarquer qu'en interview, elles mentionnent exclusivement les aspects les plus éducatifs de la fonction de juges des enfants, et passent sous silence la dimension pénale et répressive de ce poste, dont on sait pourtant par ailleurs, qu'elle est de plus en plus présente (Benec'h-Le Roux, 2008). Les magistrates transposent dans leur activité de JAF des conceptions du métier et des pratiques qu'elles se sont forgées en tant que juge des enfants. Face à la routine de la masse des dossiers, cela leur permet de ré-enchanter la fonction de JAF et de donner du sens à leur métier (Clot, 2010).

Ainsi, les magistrates appréhendent les fonctions de JAF relativement différemment de leurs homologues masculins. Toutes disent apprécier le contact avec le public, avec « les familles». Elles insistent souvent sur l'importance d'expliquer aux justiciables les points de droit qu'ils n'ont pas compris et considèrent qu'il est de leur mission d'accompagner les justiciables dans les rouages de la machine judiciaire. Une expression revient ainsi comme un leitmotiv dans la bouche d'A. Vermeer - jeune JAF de Carly qui a occupé pendant deux ans un poste de juge des enfants - pour décrire son activité : « il faut expliquer ».

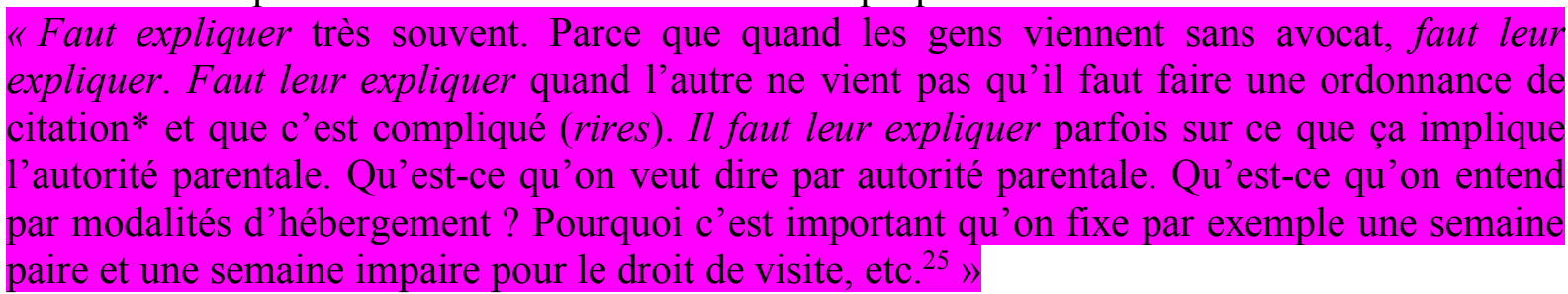

Cette posture pédagogique est particulièrement perceptible lors de l'observation des audiences de certaines magistrates. Prenons l'exemple, déjà mentionné, de la juge C. Blanchard, au TGI de Carly.

9h35. Un couple déjà divorcé demande conjointement la modification de la résidence habituelle de leurs trois enfants (âgés de 17, 15 et 13 ans) fixée jusqu'à présent chez le père. Leur fils de 13 ans habitant depuis plusieurs mois chez sa mère, ils souhaitent tous les deux officialiser leur arrangement. Au lieu d'entériner rapidement cette décision, la juge met le

\footnotetext{
${ }^{24}$ Comme le notait déjà A. Boigeol à propos des auditeurs de Justice (Boigeol, 1993, p. 516).

${ }^{25}$ Entretien avec A. Vermeer, réalisé par Jérémy Mandin, le 9 février 2009. Nous soulignons.
} 


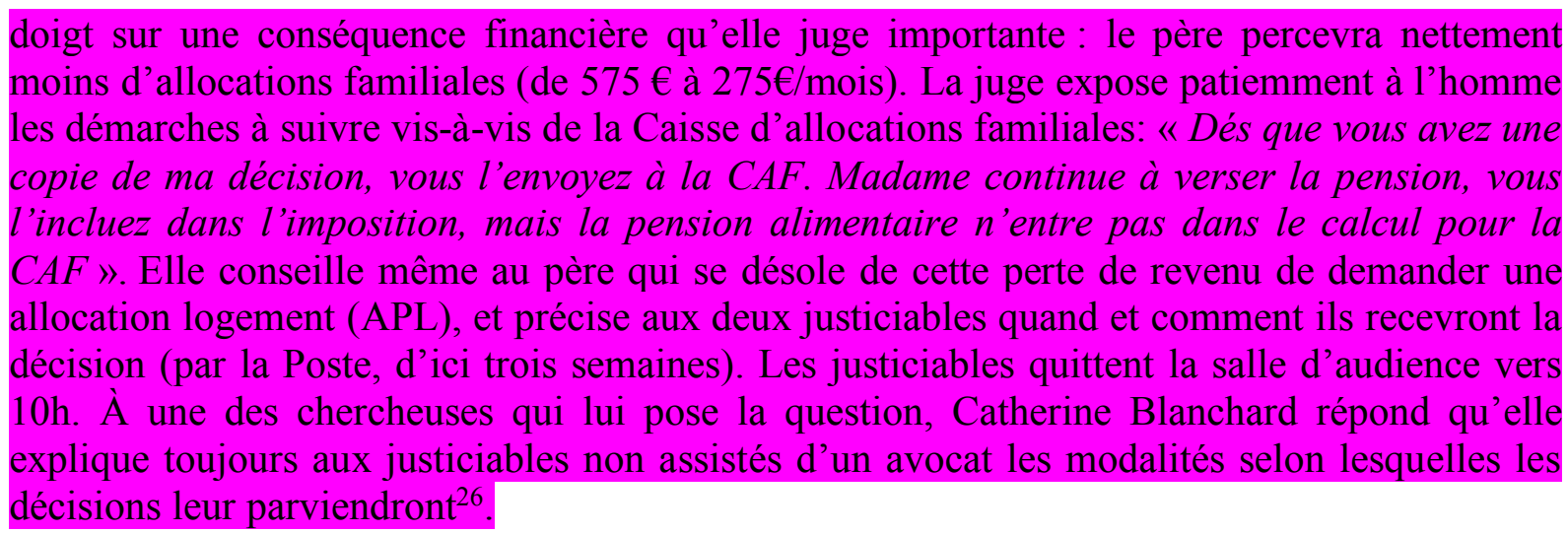

Durant cette audience, C. Blanchard a pris le temps de détailler aux deux parents toutes les conséquences pratiques - notamment financières — de leur arrangement, allant au-delà du strict cadre de la procédure judiciaire qui consiste à entériner l'accord entre les conjoints. Ce couple a passé 25 minutes devant la juge $\mathrm{C}$. Blanchard, ce qui est relativement long quand on sait que plus de la moitié des affaires que nous avons observées dans les quatre tribunaux durent moins de 13 minutes d'audience et surtout, quand on tient compte du fait que les exconjoints étaient d'accord entre eux sur tous les points avant de se présenter devant la juge ${ }^{27}$.

La posture pédagogique des femmes JAF s'accompagne d'une valorisation de la parole des justiciables. Anne-Cécile Martigue, jeune juge nommée en premier poste à Carly aux Affaires familiales et formée par C. Blanchard, explique longuement ce point: "Quand les gens signent un procès verbal d'acceptation ${ }^{28}$, je les laisse systématiquement en parler, parce que c'est le seul moment de la procédure où ils peuvent le faire [...]. C'est important qu'il y ait un tiers qui les écoute» ${ }^{29}$. Elle précise cependant que ce temps de parole reste limité par le nombre des dossiers, et surtout qu'il s'agit d'une parole hors procédure, dont elle ne tient pas compte au final dans ses décisions. La posture d'écoute et de pédagogie endossée par les femmes JAF imprègne également leur rapport à la rédaction des jugements écrits. Nombreuses sont celles insistant à cet effet sur la nécessité de "motiver » les décisions aux justiciables, afin qu'elles soient comprises et par conséquent mieux acceptées — et même si cela entre en contradiction avec des logiques d'efficacité et de productivité qu'elles ne peuvent ignorer dans un contexte global de massification du contentieux.

Les femmes juges mettent en avant l'impact de leur décision sur les existences des justiciables. Dans leur pratique du métier, que ce soit lors des audiences ou dans la rédaction de leurs jugements, elles adoptent une posture d'intervention sociale. Nous entendons par ce terme le fait qu'elles apprécient le suivi des dossiers et la recherche de solutions concrètes aux problèmes soumis par les parties. Contrairement à la plupart de leurs homologues masculins agacés par la trivialité des litiges à trancher aux Affaires familiales, plusieurs femmes JAF anciennement juge des enfants cultivent un sentiment d'utilité sociale du fait de leur intervention dans les dossiers. C'est précisément cet aspect de leur fonction qui leur procure

\footnotetext{
${ }^{26}$ Compte rendu de l'audience de la juge C. Blanchard au TGI de Carly, observée par H. Steinmetz et M. Ducret, le 11 décembre 2009.

27 Dans notre corpus d'audiences observées, parmi les 68 affaires (hors consentement mutuel) ne présentant pas de désaccord en début d'audience, seulement 7 ont duré plus de 20 minutes. Et une seule de ces 7 affaires était suivie par un homme magistrat.

${ }^{28}$ Dans les procédures de divorce autres que par consentement mutuel, la première étape est une audience de tentative de conciliation. Lors de cette audience, si les deux époux sont d'accord sur le principe du divorce et s'ils renoncent au divorce pour faute (c'est-à-dire s'ils acceptent un divorce qui ne tient pas compte des raisons de leur séparation mais ne traite que des conséquences), ils signent alors un procès verbal d'acceptation du divorce.

${ }^{29}$ Entretien avec la juge A.-C. Martigue, réalisé par J. Mandin, le 18 mai 2009.
} 
du goût pour la justice familiale.

Bénédicte le Fur a 47 ans et est JAF à mi-temps depuis 4 ans au tribunal de Valin. Elle a occupé différentes fonctions auparavant: juge d'instance, substitut du procureur et juge des enfants. Plusieurs de ses mutations ont été liées à la carrière professionnelle de son conjoint qui est ingénieur spécialisé. Elle a deux enfants en bas âge. Dans la continuité de son expérience de juge des enfants, Bénedicte Le Fur prend plaisir dans sa fonction de JAF à trouver des solutions concrètes aux problèmes des justiciables : " Je trouve qu'on peut faire des choses à l'audience, bon, pas autant qu'un juge des enfants, mais on a, malgré tout, une petite marge de manœuvre qu'on peut utiliser... et je trouve qu'il y a des décisions intéressantes à prendre et que c'est utile d'avoir été juge des enfants avant ${ }^{30} \gg$. On peut voir cette posture interventionniste à l'œuvre dans son traitement d'une affaire particulièrement « difficile», puisque les parents déjà divorcés se présentent devant le tribunal pour la neuvième fois $^{31}$ :

L'homme est ouvrier à la SNCF et la femme, ancienne employée de commerce, est au chômage. Ils sont divorcés depuis janvier 1998. La femme est à l'origine de la requête : elle demande l'autorité parentale exclusive pour sa fille âgée de 16 ans qui n'a pas vu son père depuis plus d'une année. Habituellement ce type de demande est rejetée par les JAF au nom du maintien des liens entre le père et l'enfant : l'autorité parentale conjointe est désormais la norme. Dans cette affaire, la demande de la mère est paradoxale puisqu'elle déplore l'absence de liens entre le père et sa fille. Au lieu d'entrer dans une discussion juridique sur l'autorité parentale exclusive, la juge B. Le Fur va consacrer le temps de l'audience - soit plus d'une heure - à l'analyse de la relation entre Jessica et son père. Pour ce faire, elle s'appuie sur les propos de la jeune fille recueillis lors d'une audition quelques semaines plus tôt. Jessica a dit à la juge B. Le Fur qu'elle ne « voulait plus voir son père » et a mentionné plusieurs incidents lors de leurs dernières entrevues : violences verbales à son encontre et celle de sa mère, paire de claques, interdiction du père faite à la jeune fille d'avoir des contacts avec sa famille et notamment avec l'enfant qu'il a eu avec sa nouvelle compagne. Ce dernier point est au centre de l'audience avec les parents. La juge B. Le Fur sermonne l'homme : « Vous êtes un adulte responsable. Jessica est remontée contre vous, mais pas contre tous les membres de votre famille. Et vous, vous avez du poids par rapport à votre famille ! [...] C'est dramatique, Jessica est coupée de son papa et de toute sa famille, elle n'a jamais vu son frère, le fossé se creuse et deviendra bientôt un précipice, c'est aberrant que dans le monde où l'on vit elle n'ait jamais vu son frère ! ». L'homme reste sur ses positions et refuse de s'excuser auprès de sa fille. Devant la juge, les deux parents se disputent, la mère suppliant le père de reprendre contact avec Jessica. La juge conclut l'audience en s'adressant à l'homme : " vous avez fait beaucoup de dégâts, c'est dommage. Il ne faut pas être rigide sur ses positions. Moi, je ne peux pas vivre à votre place. Votre fille a besoin de vous et de toute votre famille ». Son jugement du 25 mars 2010 accorde l'autorité parentale exclusive à la mère, tout en ouvrant la possibilité d'une modification de cette décision en cas de rapprochement entre le père et sa fille.

Dans cette affaire, la juge B. Le Fur utilise ce qu'elle appelle les « marges de manœuvre » de la procédure - entrevues répétées avec les parents et leurs avocats, long dialogue avec les parties lors de l'audience, audition de l'enfant — pour tenter d'améliorer les relations entre un père et sa fille adolescente. Sa décision devient en elle-même un instrument pédagogique : fixer une autorité parentale exclusive est conçu comme une manière de faire comprendre au

\footnotetext{
${ }^{30}$ Entretien avec B. Le Fur, réalisé par Céline Bessière et Benoît Coquard, le 4 mars 2010.

${ }^{31}$ Observation de l'audience de la juge B. Le Fur au tribunal de Valin réalisée par C. Bessière et B. Coquard, le $1^{\mathrm{er}}$ mars 2010, et de l'analyse du dossier, notamment le compte-rendu de l'audition d'enfant du 14 janvier 2010 et le jugement du 25 mars 2010.
} 
père qu'il doit reprendre contact avec sa fille. C'est sans doute ce type de décision exceptionnelle - à rebours de l'interprétation juridique commune puisque habituellement l'autorité parentale exclusive vise à protéger l'enfant de l'influence de l'un de ses parents qu'elle qualifie de "décisions intéressantes » dans l'entretien cité plus haut. Remarquons cependant que lorsqu'un magistrat n'est plus seulement pédagogue du droit, mais également "pédagogue de la famille», son attitude interventionniste se double d'une posture moralisatrice vis-à-vis des parents (ici le père).

B. Le Fur défend sa conception interventionniste aux Affaires familiales par contraste avec d'autres collègues qu'elle estime plus « passifs » qu'elle sur les dossiers : " Moi ce que je détesterais, si vous voulez, ça serait être la chambre d'enregistrement, ne rien pouvoir dire, ne pas pouvoir intervenir, interpeller les gens, prendre ce qu'on me donne et me démerder avec. Je pense qu'il y a des collègues au contraire qui sont très contents de faire comme ça, à mon avis... Je pense que ce ne sont pas les meilleurs d'entre nous $»^{32}$. Nous allons voir à présent que la posture qu'elle dénonce ici est revendiquée par des magistrats hommes qui promeuvent une conception « aseptisée » de la fonction de juge aux Affaires familiales et sont au contraire rebutés par son caractère potentiellement intrusif. Pour comprendre ces différences de conception du poste entre les JAF hommes et femmes, il est nécessaire de les réinscrire dans le cadre d'ensemble de leur trajectoire familiale et professionnelle.

\section{En passant par le JAF : la carrière des juges hommes}

Les hommes et les femmes juges n'arrivent pas aux Affaires familiales au même moment de leur carrière et de ce fait n'y n'occupent pas les mêmes positions. Nous avons réalisé des entretiens enregistrés avec l'ensemble des huit hommes JAF des quatre tribunaux enquêtés. Quatre ont le statut de vice-président (VP), c'est-à-dire une fonction de premier grade ${ }^{33}$. À titre de comparaison, dans les quatre tribunaux de l'enquête, au début de l'année 2009, sur 20 femmes JAF, trois seulement étaient vice-présidentes. Cette surreprésentation des hommes en haut de la hiérarchie n'est pas spécifique aux Affaires familiales. Les données du ministère de la Justice indiquent qu'en 2011 les femmes représentent les trois quarts des juges du second grade, un peu plus de la moitié des effectifs du premier grade, mais moins d'un tiers des effectifs hors-hiérarchie. Ainsi, plus on grimpe dans la hiérarchie de la magistrature, plus les hommes sont nombreux ${ }^{34}$. Par ailleurs, alors que l'entrée dans la carrière des magistrates se faisait dans la foulée de leurs études de droit, par le premier concours d'entrée de l'ENM, les hommes JAF que nous avons rencontrés sont plus âgés et ont des carrières moins linéaires. Cinq parmi les huit hommes JAF de notre enquête sont entrés tardivement dans la magistrature: pour deux d'entre eux après des postes dans d'autres administrations (au

\footnotetext{
${ }^{32}$ Entretien avec B. Le Fur, femme, 47 ans, JAF au tribunal de Valin, réalisé par C. Bessière et B. Coquard, le 4 mars 2010.

${ }^{33}$ Les juges débutent leur carrière au premier échelon du second grade. Le second grade comporte cinq échelons et le passage d'un échelon à l'autre s'effectue à l'ancienneté. Le passage du second au premier grade est précédé par l'inscription à un tableau d'avancement dressé par une commission indépendante. Il nécessite sept ans d'exercice professionnel, dont cinq ans au moins en qualité de magistrat dans une juridiction ou l'administration centrale du ministère de la Justice. Le passage en hors-hiérarchie des juges du siège s'effectue au choix de l'autorité de nomination (le président de la République) avec avis conforme du Conseil supérieur de la magistrature.

${ }^{34}$ La surreprésentation des hommes en haut de la hiérarchie de la magistrature s'explique en partie par un effet de génération. Du fait de l'avancement de la carrière par ancienneté, on retrouve en bas de l'échelle les jeunes générations de juges très féminisées. Mais la féminisation de la Justice étant un processus enclenché depuis plus de 40 ans, on peut en conclure que la magistrature judiciaire n'échappe pas au phénomène du "plafond de verre » qui limite l'accès des femmes aux postes de direction les plus prestigieux (Laufer, 2004).
} 
ministère des Affaires sociales et dans le secteur médico-social), pour les autres après une première carrière dans d'autres métiers du droit (un ancien avocat, un ancien juriste d'entreprise, un ancien enseignant à la Faculté de droit). Ces juges aux carrières atypiques décrivent leur arrivée aux Affaires familiales comme le fruit d'un hasard, lié aux contingences organisationnelles des tribunaux.

Étienne Paletot, par exemple, a intégré la magistrature à 48 ans à la suite d'une première carrière d'enseignant à l'École nationale des Greffes et à la Faculté de droit. Il était juge d'instance depuis quelques années dans une petite juridiction, quand celle-ci a été supprimée par la réforme de la carte judiciaire. "Contraint et forcé », il a dû demander sa mutation au TGI de Marjac, la ville où il réside, et s'est retrouvé malgré lui affecté aux Affaires familiales. Quelques jours après cette affectation, il a été promu au premier grade. Il raconte qu'il a alors immédiatement «téléphoné à la Chancellerie » (c'est-à-dire au ministère de la Justice) pour négocier un changement de fonction. Sa hiérarchie lui a fortement conseillé d'accepter le poste de JAF : "si vous refusez maintenant, vous vous grillez pour l'avenir pour nous ", rapporte-t-il. Six mois plus tard, il a déjà entamé les démarches pour quitter les Affaires familiales : «tout sauf le JAF, ça devient ça, moi, en ce moment ! » avoue-t-i1 ${ }^{35}$. On peut relier ce rejet fort des Affaires familiales et de leur dimension pratique à la trajectoire de ce juge, qui a enseigné et étudié longuement le droit (il a ainsi commencé une thèse dans cette discipline). Après quelques années, il se lasse de l'enseignement et collabore avec la Cour de cassation dans le cadre d'une mission temporaire. Il refuse le poste à plein temps qu'on lui offre pour rester dans sa région d'habitation, et passe alors le concours pour entrer dans la magistrature. Il explique en entretien combien il a trouvé passionnant ce travail à la Cour de cassation « où l'on ne fait que du droit en fait ». La distance est alors grande entre la pratique quotidienne du droit telle qu'elle s'exerce aux Affaires familiales et ses aspirations initiales.

Rien ne prédisposait non plus le juge Georges Kawl à devenir JAF au TGI de Carly en septembre 2006. Né en 1952, ancien juriste pour un syndicat de producteurs de cinéma pendant près de 20 ans, il a lui aussi commencé un doctorat en droit. Après un licenciement économique, il passe le " concours d'entrée exceptionnel » pour entrer dans la magistrature en 2000 avec le projet de devenir conseiller à la Cour d'appel. Il occupe ce poste dans un tribunal du Sud-Ouest pendant quelques années. Promu au premier grade en 2006, il aurait souhaité devenir premier conseiller dans une Cour d'appel, mais cherche également à se rapprocher de Paris où il n'a pas cessé d'habiter. Il obtient alors le TGI de Carly, dans la grande couronne. Le président du tribunal de Carly lui propose de devenir juge des libertés et détention (un poste réservé aux vice-présidents) qui correspond bien à son expérience en correctionnelle. Bien qu'il trouve le contentieux intéressant, G. Kawl refuse le poste du fait de l'incertitude des horaires et des permanences nombreuses le soir et le week-end ( JLD, Jamais Libre pour Dîner ! »), alors qu'il n'a pas de voiture et dépend des transports en commun pour se rendre à Carly. Il prend donc le poste de JAF : « Le président du TGI était sûr que j’allais prendre JLD parce qu'Affaires familiales, j'avais dû lui dire que ça ne me plaisait pas. Enfin c'est vrai que ce n'est pas très juridique. J'aimerais bien faire un peu de droit moi quand même! (petit rire) Et donc j'ai pris JAF pour ces raisons pratiques tout bêtement ». Ces deux exemples rejoignent des propos entendus chez d'autres magistrats hommes. Ceux-ci ont eu pour ambition première d'occuper des fonctions marquées par leur caractère plus prestigieux et aspirent souvent à occuper des postes dans des juridictions de seconde instance, moins en première ligne du traitement de la masse des affaires.

\subsection{Frustrations et dégoûts}

\footnotetext{
${ }^{35}$ Entretien réalisé par C. Bessière et Aurélie Fillod-Chabaud, le 19 fevrier 2009.
} 
Comme celles des femmes, les carrières des juges hommes sont tributaires des logiques de l'institution judiciaire, et de la disponibilité des postes. De même, beaucoup de magistrats cherchent à limiter les coûts de la mobilité géographique requise par leur profession. En revanche, leur perception de la fonction semble opposée : alors que les femmes se disent plutôt satisfaites de leur poste, plusieurs hommes expriment leur frustration, voire leur souhait d'en partir le plus rapidement possible, pour accéder à des postes plus prestigieux.

Ainsi, le juge Pierre Terreau, après une longue carrière de juge d'instruction est devenu président de la Chambre de la famille au TGI de Marjac. En poste depuis six mois quand nous le rencontrons pour la première fois, il décrit en riant son arrivée aux Affaires familiales en multipliant les bons mots «au bout de 22 ans de métier, c'est comme si on passait de ramoneur à égoutier ", "je suis passé d'un service où on faisait de la haute couture à un service où l'on fait du prêt-à-porter chinois $»^{36}$. Le juge $P$. Terreau a du mal à cacher son manque d'enthousiasme face à la fonction de JAF : il aurait préféré de loin une affectation au Pénal. Il aspire à être président de Cour d'assises et envisage dans ce but un passage par la Cour d'appel. Pour ce faire, il doit rester au minimum deux ans sur un poste de vice-président généraliste comme celui qu'il occupe actuellement, mais il pourrait accepter entre temps un poste de vice-président en correctionnelle au TGI de Marjac. À plusieurs reprises au cours de l'enquête, il nous lance comme une boutade qu'il ne sera bientôt plus JAF : "j'espère être parti avant d'être un très bon JAF!", «je me suis égaré au JAF, mais pas pour très longtemps!».

Par contraste avec leurs collègues femmes qui décrivent au cours des entretiens leur intérêt et leur goût pour les Affaires familiales, les hommes JAF profitent de la présence des sociologues pour exprimer leurs réserves, leurs problèmes, voire leur dégoût pour cette fonction. Jean Brunetti est le président de la Chambre de la famille à Valin, un gros tribunal situé dans une grande ville de province. En janvier 2009, face aux difficultés d'engorgement de certains cabinets et aux nombreux retards, J. Brunetti a été nommé à la tête des Affaires familiales en raison de sa longue expérience : il est spécialement chargé par le président du tribunal de redessiner l'organisation de la Chambre. Âgé d'une cinquantaine d'années, il a occupé de nombreuses fonctions depuis sa sortie de l'ENM en 1987 (juge d'instruction, juge à la Cour d'assise, juge à la Cour d'appel, secrétaire général du tribunal de Valin, etc.). Il décrit tous ses passages aux Affaires familiales comme des passages obligés : une première fois, lors de son arrivée au TGI de Valin de 1998 à 2002 (" quand on arrive à Valin, on atterrit toujours au JAF ! »), une deuxième fois, brièvement, en tant que vice-président en 2004, («j'ai réussi à partir assez vite à mi-temps, puis complètement $\gg)^{37}$. Il a plus particulièrement apprécié par contraste son passage en Cour d'assise où il a travaillé sur des procédures pénales en chambre d'accusation, mettant en avant l'aspect « juridique pur » de cette tâche, mais aussi le fait de travailler sur des affaires aux dimensions parfois politiques ou financières et où l'urgence porte sur des enjeux qu'il estime plus importants. J. Brunetti ne cache pas qu'à nouveau, en 2009, il a pris la fonction de président de la Chambre de la famille à contrecœur. Dans une situation plus informelle — dans le hall d'entrée des Affaires familiales, à côté de la machine à café, juste après le déjeuner - il se plaint plus explicitement du contenu de son travail. " JAF c'est vraiment le pire », " cette haine dans les couples, elle nous tue, elle nous épuise, je n'en peux plus! ». Le juge Brunetti se plaint que les justiciables utilisent l'audience pour se disputer entre eux, qu'il aurait envie de leur dire parfois d'aller prendre un café et de discuter, qu'il n'a pas besoin d'assister à cela ${ }^{38}$.

Alors que les femmes JAF qui ont été juges des enfants considèrent leur fonction comme

\footnotetext{
${ }^{36}$ Entretien avec le juge Terreau, réalisé par Emilie Biland et Pierre de Larminat, le 17 février 2009.

${ }^{37}$ Informations recueillies par Aurélie Fillod-Chabaud et Benjamin Faure, le 3 mars 2010.

${ }^{38}$ Informations recueillies par C. Bessière, le 2 mars 2010.
} 
moins «stressante », plus compatible avec des charges familiales, les hommes vivent plus difficilement la masse et la répétitivité du contentieux. Ceci est à relier avec ce qui déplaît fortement aux hommes JAF : supporter la tension émotionnelle entre les conjoints lors des audiences.

\subsection{Des difficultés face à l'intimité des justiciables}

Les juges hommes rencontrés expriment souvent une forte réticence à entrer dans l'intimité des justiciables. Certains, comme le juge É. Paletot au TGI de Marjac, n'ont pas de mot assez fort pour décrire toute l'aversion qu'ils ont pour cette fonction : un contentieux « déprimant », « terrible », « dévastateur ${ }^{39}$. Au milieu de la cinquantaine, civiliste de formation, ancien enseignant, il rêve de la Cour de cassation ou d'un poste de juge dans une chambre civile qui ne ferait que du droit de la construction, sans contact avec les justiciables. Il se dit même prêt à occuper un poste de juge d'application des peines, une fonction éprouvante mais qui lui permettrait de couper davantage avec sa vie personnelle : «c'est une réalité qui est marginale, ceux qui sont en prison, c'est pas la norme. Là, le JAF, ça vous renvoie à une image de la société qui est une image courante, ça renvoie à une image du couple qui est un peu dérangeante. Il y a des fois, on disjoncte, je vous assure ! » Cette comparaison entre juge d'application des peines et JAF est intéressante car elle met bien l'accent sur la difficulté des juges hommes à se positionner dans les contentieux familiaux (et le peu d'attrait pour le traitement des affaires « courantes »).

Mettant en avant son goût pour le contenu juridique des affaires, le juge É. Paletot exprime une aversion pour les Affaires familiales en tant qu'elles le font entrer bien plus qu'il ne le souhaiterait dans l'intimité des justiciables :

"C'est très désagréable, je vais vous dire, que moi dès fois, je suis mal à l'aise. Bon j'essaye
d'en plaisanter parce que ma nature c'est de traiter les choses avec dérision [...]. Mais j'ai
l'impression d'être, pour parler vulgairement, de mater quoi, c'est du (il hésite) voyeurisme
[...]. Il y a des choses que je n'ai pas envie de savoir, en fait! Moi j'aime le droit parce que
c'est une vocation. J'aime la règle de droit, j'aime la spéculation juridique. Mais j'ai pas envie
de savoir si Monsieur est allé coucher avec Madame [...]. Mais si vous voulez, il leur faut un
arbitre. Et voilà, donc on est là pour essayer de trouver des solutions. Mais je pense que ça
pourrait être fait par n'importe quelle personne. Il n'y a pas besoin d'être juriste pour ça !40»

Là où les juges femmes disent aimer faire preuve d'inventivité pour trouver des solutions concrètes aux problèmes des justiciables, les hommes JAF sont davantage réticents à entrer dans les détails des affaires privées. Ils sont particulièrement rebutés par les questions d'horaires - horaires d'école, de crèche, de travail, de bus - de calendriers, les calculs de montants de pension alimentaire. Ils considèrent que tous ces aspects pratiques devraient êtres réglés entre les parties, sans passer par un magistrat. Ainsi, les propos des hommes JAF contrastent nettement avec la posture pédagogique et interventionniste des femmes JAF. Le juge É. Paletot estime avec un brin de désespoir que le JAF fait un travail proche de celui des assistantes sociales avec « des parties à qui il faut tout expliquer ». Il reconnaît faire appel à sa greffière (« en plus c'est une femme, on n'a pas du tout la même approche, c'est intéressant d'avoir une approche de femme») lorsque les questions pratiques dépassent ses compétences :

« elle m'aide parce que, bon moi je n'ai plus d'enfants petits. Donc c'est vrai que les
contingences matérielles maintenant ça s'est un petit peu éloigné. Donc tout ce qui est
matériel ou pratique, elle me dit ben oui vous savez, y a pas de bus à cette heure-là... bon

\footnotetext{
${ }^{39}$ Entretien avec le juge É. Paletot, réalisé par C. Bessière et A. Fillod-Chabaud, le 19 février 2009.

40 Ibid.
} 


\section{voilà... heureusement qu'elle est là parce que... ça me facilite la vie ! ».}

De la même façon, le juge Emile Fratta qui occupe son premier poste en tant que JAF à Carly déplore d'avoir à se prononcer sur des questions d'horaire de garderie ou de partage des vacances scolaires. Il soupire : «Voilà ce à quoi le juge, il est ramené à faire ! ${ }^{41}$ ». Il regrette que les JAF aient à se prononcer sur de tels litiges qu'il considère comme triviaux et qui rabaissent la fonction de magistrat.

On retrouve ici le regret d'une banalisation de la fonction de juge, d'une dilution de son autorité dans le contentieux de masse, avec des magistrats amenés à gérer "l'urgence du quotidien » (Boigeol, 1997, p. 27). Présentant le rôle du juge comme un arbitre au-dessus de la mêlée, les hommes JAF n'apprécient ni l'intrusion dans l'intimité des justiciables, ni d'être sollicités pour des questions pratiques qu'ils considèrent comme n'étant pas de leur ressort. C'est pour ces raisons que les hommes JAF se plaignent davantage que leurs collègues féminines de la masse du contentieux, qui leur paraît répétitif et ennuyeux, loin de leurs compétences et de leur intérêt pour le droit. Mais les hommes JAF ne se contentent pas d'exprimer des critiques et un certain dégoût pour leur fonction. Ils développent également leur propre conception "idéale" de la fonction, en prenant le contre-pied des pratiques interventionnistes de leurs collègues que nous avons décrites plus haut et qu'ils considèrent comme «trop moralisantes ». En reprenant les mots du juge Pierre Terreau, on peut qualifier cette vision masculine d'une conception « aseptisée » de la fonction de JAF.

P. Terreau est président de la Chambre de la famille au tribunal de Marjac, à la suite d'une longue carrière de juge d'instruction. Au cours d'une audience, entre deux affaires, P. Terreau montre sa calculatrice aux sociologues d'un air fatigué et en commentant : «ça, c'est l'outil du JAF, et non pas le Code!». Selon lui, «fixer une pension alimentaire, ce n'est pas du droit $!^{42} \gg$ Cette proposition est particulièrement provocante quand on sait que plus de la moitié des litiges aux Affaires familiales portent sur cette question. Il nuance alors son propos : «fixer le principe d'une pension alimentaire c'est du droit. Fixer le montant ce n'est pas du droit ». Pour cet ancien juge d'instruction, les calculs de pension alimentaire sont une tâche ingrate fort éloignée de ce qui fait la spécificité de la profession de magistrat, à savoir l'habileté à mobiliser à bon escient des savoirs juridiques complexes et spécialisés, dans des situations toujours particulières (Champy, 2009). Le juge P. Terreau déplore le fait que les JAF comblent « la carence des époux qui n'arrivent pas à se mettre d'accord » et il développe une vision de la fonction fondée sur le refus de l'intrusion dans les vies privées des justiciables et la prime à l'accord des conjoints : "Moi j'ai une conception très, je dirais très aseptisée de la fonction. Le juge n'est pas là pour faire la morale ou pour donner des leçons aux gens. Il peut attirer l'attention sur telle difficulté, sur le fait que les choses ont évolué, voilà, mais en étant très neutre et sans donner de leçon ». Ainsi, comme l'atteste l'observation d'audience suivante, le juge $\mathrm{P}$. Terreau tient à distance les parties en ne posant que les questions strictement nécessaires au règlement de la procédure, en cadrant les moments de débordements émotionnels, en cherchant à entrer aussi peu que possible dans l'histoire des justiciables et surtout en privilégiant l'accord — coûte que coûte — entre conjoints.

Deux parents non mariés se présentent devant le juge P. Terreau, au sujet de leur bébé âgé de
huit mois. Le juge demande où habite l'enfant. La femme répond: "chez moi, avec ses deux
sœurs ». Le juge hésite un instant, vous voulez dire « demi-sœurs »; elle répond : " oui, c'est
un autre papa ». L'homme précise qu'ils n'ont jamais vécu ensemble et ajoute de façon
solennelle et maladroite: " J'aimerais me déchoir de mes droits parental[s] ». Le juge
reformule : "vous voulez dire que vous voulez seulement l'autorité parentale pour la mère»,

\footnotetext{
${ }^{41}$ Entretien avec le juge E. Fratta, réalisé par J. Mandin et H. Steinmetz, le 27 janvier 2009.

${ }^{42}$ Entretien avec le juge P. Terreau, réalisé par E. Biland et P. de Larminat, le 17 février 2009.
} 
il répond: " oui, on s'est consultés, on est d'accord ». Le juge s'interrompt : " Je dois vous demander des explications, parce que ce n'est pas ce qu'on fait habituellement ». Le père explique qu'il a reconnu l'enfant mais ne l'a jamais vu. [...] Il faut dire que c'est plus un accident qu'autre chose, tout en sachant que j'ai déjà deux filles ». Le juge P. Terreau conclut rapidement : « vous connaissez mieux la situation que moi ». Il demande s'ils souhaitent « un droit de visite à l'amiable ». « Est-ce qu'on homologue ça, ou vous proposez autre chose ? ». Les parents paraissent perplexes mais finissent par acquiescer. Enfin, le juge indique que la requête déposée par la femme demande une pension alimentaire mensuelle de $160 €$. Malgré les relances du juge, la mère ne défend pas oralement sa demande, que le père récuse « $160 €$, c'est pas la peine, c'est ce que je donne pour mes deux [autres] filles », j'ai un revenu de $1000 €$ par mois ». P. Terreau suggère : « il faut peut-être partager la masse en trois, et réduire la pension de vos premiers enfants. Soit on rentre dans le détail, soit vous vous mettez d'accord ». L'homme commence à chuchoter à l'oreille de son ex-conjointe. Le juge leur propose de sortir pour régler cela ensemble.

Les justiciables sortis, le juge s'interroge sur la nature de leur relation («adultérine»?) et s'étonne qu'ils n'aient pas discuté de la pension pendant l'attente avant l'audience. Pendant ce temps, dans la salle des pas perdus, la femme a l'air éprouvée. Au fur et à mesure de la discussion, l'homme s'énerve, sans toutefois hausser la voix. La femme est adossée contre le mur. Bien plus grand qu'elle, l'homme lui barre le passage. Un quart d'heure après la suspension, les parents entrent à nouveau dans la salle d'audience. À peine assis, la femme annonce qu'ils sont tombés d'accord sur un montant de $60 € /$ mois. Le juge entérine la décision sans rien ajouter. Une fois les justiciables sortis, le magistrat commente laconiquement : «Il négocie mieux qu'elle ! » ${ }^{43}$

La pratique des audiences du juge P. Terreau est en accord avec sa conception " aseptisée » de la fonction de JAF. Dans cette audience difficile, où le juge soupçonne une relation adultérine et secrète entre les parties ("il a peut-être quelqu'un par ailleurs qui n'est pas au courant » dit-il aux sociologues), il ne cherche pas à du tout à entrer dans l'histoire des justiciables, ni à proposer une conception de la co-parentalité, à convaincre le père de s'investir auprès de son enfant. P. Terreau ne pose que les questions strictement nécessaires au règlement de la procédure - la justification de l'autorité parentale à la mère — et il entérine l'accord entre les parties y compris lorsqu'elles sont le résultat d'un rapport de force, ici observable physiquement dans la salle des pas perdus. C'est l'intrusion dans des affaires « ordinaires » qui pose problème à cet ancien juge d'instruction qui a eu l'occasion pourtant d'ordonner des enquêtes poussées dans l'intimité des justiciables dans le cadre de sa précédente fonction, mais le fait d'intervenir dans cette intimité par ses décisions.

\subsection{Une vision négative commune du poste, mais des pratiques différentes : style " aseptisé » vs. style "provocateur»}

Tous les magistrats qui envisagent les Affaires familiales comme une étape obligée mais peu gratifiante de leur carrière n'adoptent pas cette manière de conduire l'audience. É. Paletot, collègue de $\mathrm{P}$. Terreau à Marjac, éprouve lui aussi, nous l'avons vu, des difficultés face à l'exposition de l'intimité des justiciables. Mais loin de la pratique aseptisée de son collègue, il pratique selon ses propres termes «l'humour et la dérision" pour mettre à distance les problèmes des justiciables. Ainsi, il ne se prive pas de distribuer bons mots, plaisanteries et commentaires (parfois désagréables) aux parties.

Lors d'une audience, il questionne et sermonne les parents d'enfants en bas âge : «c'est

${ }^{43}$ Extrait du compte-rendu d'observation de l'audience du juge P. Terreau au tribunal de Marjac, réalisé par C. Bessière et Shahideh Noorolahian Mohajes, le 18 février 2009. Observation en parallèle dans la salle des pas perdus effectuée par Sibylle Gollac et Raphaëlle Salem. 
étonnant d'avoir des enfants et de se séparer dans les mois qui suivent ? », ajoutant : "Vous vous séparez quatre mois après avoir acheté une maison ensemble. Dans l'ordre de l'inconséquence, on ne fait quand même pas mieux! ». Un peu plus tard, il s'adresse à un père qui refuse de payer une pension alimentaire : " personne ne vous a obligé ni à avoir des relations sexuelles, ni à avoir des enfants $\gg{ }^{44}$.

La pratique d'É. Paletot montre qu'il existe plusieurs manières de faire face aux difficultés éprouvées dans la fonction. Elle nous garde aussi de considérer que seules les magistrates adoptent un registre moralisateur. Le second degré pratiqué par ce juge fait affleurer de nombreux jugements de valeurs (sur la sexualité des conjoints ou l'éducation des enfants) qui peuvent heurter les justiciables, sommés de se raconter pour se justifier. L'attitude d'É. Paletot lors des audiences paraît alors provocatrice et inquisitrice, aux antipodes à la fois du style aseptisé mis en avant dans les entretiens par les juges hommes (dont le juge É. Paletot lui-même) et du style pédagogique pratiqué par les juges femmes.

\section{Des exceptions qui confirment la règle : la construction sociale du genre des juges}

Les deux carrières typiques de magistrates et magistrats aux Affaires familiales que nous avons dégagées permettent de rendre compte d'une grande partie des pratiques et des conceptions de la fonction de JAF. Dans le cas des femmes juges, la socialisation primaire féminine au souci des autres (source de leur vocation précoce pour la fonction de juges des enfants) a été renforcée par des fonctions professionnelles où ce souci est le plus légitime : la posture interventionniste des femmes JAF se rapproche du travail du care, autrement dit des activités d'aide à autrui qui allient compétences techniques (ici juridiques) et émotionnelles (Hochschild, 1983). Du côté de leurs collègues masculins, les attitudes se polarisent entre un registre procédural conforme à la neutralité attendue des fonctionnaires et un registre provocateur, qui n'est pas sans rappeler l'humour viril pratiqué par les médecins (Zolesio, 2009) et les policiers (Pruvost, 2007, pp. 259-261). La position des juges hommes rejoint une distribution genrée des spécialités analysée par A. Boigeol, les magistrats valorisant la confrontation avec le maintien de l'ordre public, la politique, et laissant aux femmes les « fonctions sociales de contact avec les enfants et les familles, les services sociaux » (Boigeol, 1997, p. 31).

Néanmoins, toutes les femmes JAF n'apprécient pas les Affaires familiales autant que les anciennes juges des enfants et tous les hommes JAF ne sont pas autant rebutés par la fonction. L'analyse des cas suivants - qui s'écartent sensiblement des deux modèles féminin et masculin d'occupation de la fonction de JAF — nous permet de mesurer combien ce n'est pas en soi le fait d'être un homme ou une femme qui contribue à des conceptions et des pratiques différentes du métier de JAF, mais qu'il s'agit d'une construction sociale du genre des juges, qui se joue tout au long de leur trajectoire familiale, scolaire et professionnelle.

\subsection{Des femmes juges qui n'aiment pas les Affaires familiales}

Certaines magistrates rencontrées ne s'inscrivent pas dans le modèle typique des carrières féminines décrit plus haut. N'étant pas passées par la fonction de juge des enfants, ayant effectué des carrières en correctionnelle ou au Parquet, elles ont en commun avec les hommes de passer un peu par hasard par le JAF et partagent avec ces derniers une vision dévalorisée de la fonction. Elles perçoivent les Affaires familiales comme une tâche ingrate et monotone et cherchent à écourter au maximum leur passage dans ce poste.

\footnotetext{
${ }^{44}$ Extrait du compte-rendu d'observation de l'audience du juge É. Paletot au tribunal de Marjac, réalisé par S. Gollac et R. Salem, le 19 février 2009.
} 
Martine Parnasse, âgée d'une cinquantaine d'années, est JAF depuis quelques mois avec le grade de vice-présidente au tribunal de Carly ${ }^{45}$. Venant d'une famille de magistrats (son père a fini sa carrière comme juge en Cour de cassation, trois de ses six frères sont eux aussi dans la magistrature), elle a derrière elle une longue carrière de juge, où elle a occupé longtemps et souvent des fonctions en correctionnelle. Elle a déjà exercé la fonction de JAF entre 1983 et 1986. Elle dit ne pas avoir accordé beaucoup d'importance à l'avancement dans sa trajectoire professionnelle : en effet, après un début de carrière dans un petit tribunal d'une zone rurale, elle a choisi le tribunal de Carly dans les années 1980 afin de se rapprocher du lieu de travail de son mari, ancien ingénieur, professeur dans une grande école scientifique parisienne. M. Parnasse n'apprécie pas du tout le travail aux Affaires familiales : " Ca ne me réussit pas » dit-elle, et, alors même qu'elle vient de prendre le poste, elle pense déjà à sa prochaine mutation à l'application des peines (un des postes pourtant les moins prisés). Le rejet de la fonction passe par la critique des conditions de travail. Plus âgée que la plupart des femmes rencontrées, ses enfants sont déjà grands (l'un est élève de l'Ecole normale supérieure, l'autre est au lycée), elle ne valorise pas comme les autres femmes le fait de pouvoir rentrer tôt chez elle. De plus, ne possédant pas d'ordinateur personnel, et ne maîtrisant par l'informatique, elle déteste le travail de rédaction des décisions que la fonction de JAF implique.

Âgée d'une quarantaine d'années au moment de l'entretien, la juge Valentine Langlade partage des caractéristiques communes avec les autres femmes JAF (une vocation précoce, un excellent parcours scolaire), mais placée aux Affaires familiales du tribunal de Valin dans le cadre d'un contrat d'objectif visant à désengorger un cabinet "planté » par la masse des dossiers en attente, elle n'apprécie pas particulièrement la fonction : «JAF, ça me sort par les yeux ! ${ }^{46}$. Elle souligne surtout la monotonie du contentieux et la pression pour faire baisser le stock des dossiers dans son cabinet. Elle juge la fonction trop spécialisée et donc ennuyeuse en comparaison avec les fonctions de juge d'instance dans des petites juridictions qu'elle a occupées auparavant. En poste depuis février 2009 (après la naissance de son premier enfant), elle quitte le JAF en septembre 2010 pour devenir vice-présidente dans un tribunal d'une autre région. Cette mutation est le résultat d'un choix personnel et familial puisqu'elle souhaite quitter Valin pour s'installer avec son mari et sa fille à plein temps dans leur maison de campagne. " J'ai pas un parcours logique de carrière » commente-t-elle.

Ainsi ces deux femmes JAF ont en commun avec les autres magistrates l'influence de leur trajectoire familiale dans l'avancement de leur carrière. En revanche, contrairement aux anciennes juges des enfants, elles ont plutôt une vision négative du poste. Comme pour les juges hommes, la fonction de JAF constitue une étape peu appréciée de leur carrière. Elles invoquent cependant davantage la monotonie de la tâche et les conditions de travail qu'un rejet de l'intrusion dans l'intimité des justiciables.

\subsection{Une vision militante des Affaires familiales : la carrière atypique du juge Yves Defert}

Par contraste, un homme, parmi les huit juges que nous avons rencontrés, a longtemps occupé un emploi d'infirmier (un emploi du secteur du care par excellence, majoritairement occupé par des femmes), et se distancie fortement du modèle masculin décrit plus haut. Yves Defert $\mathrm{du}$ tribunal de Belles pense ainsi sa fonction sur le mode de l'intervention sociale. Il s'intéresse beaucoup aux relations parents-enfants et estime que la justice familiale a un rôle essentiel à jouer dans la prévention des «dysfonctionnements » familiaux et même de la «folie $»^{47}$. Y. Defert renverse l'image dévalorisée des Affaires familiales dans les TGI. Il considère que les Affaires familiales sont une « affaire sérieuse », une «branche essentielle

\footnotetext{
${ }^{45}$ Entretien réalisé par Samuel Bizien, le 25 janvier 2011.

${ }^{46}$ Entretien réalisé par Samuel Neuberg, le $1^{\text {er }}$ Mars 2010

${ }^{47}$ Entretien avec le juge Y. Defert, réalisé par E. Biland et J. Mandin, le 20 mars 2009.
} 
du droit » qui « prend le mal à la racine » (par opposition à la justice pénale). Il regrette ainsi que les Affaires familiales soient autant négligées par l'institution judiciaire, et dénigrées par ses collègues. Cette manière de considérer les relations familiales comme essentielles s'ancre dans une trajectoire professionnelle atypique. Comme d'autres juges hommes rencontrés, Y. Defert a passé le concours de la magistrature en admission parallèle à 35 ans passés. Moins habituel pour un juge est le domaine dans lequel il a exercé sa première carrière professionnelle : 15 ans dans le secteur médico-social. Aujourd'hui, à l'âge de 45 ans, ses prises de positions et ses pratiques de magistrat (d'abord en tant que juge d'application des peines, puis JAF) sont marquées par son parcours antérieur. Considérant que les juges sont mal formés dans cette discipline à l'ENM, il met en avant les compétences relationnelles héritées de son ancien métier qu'il considère comme primordiales aux Affaires familiales :

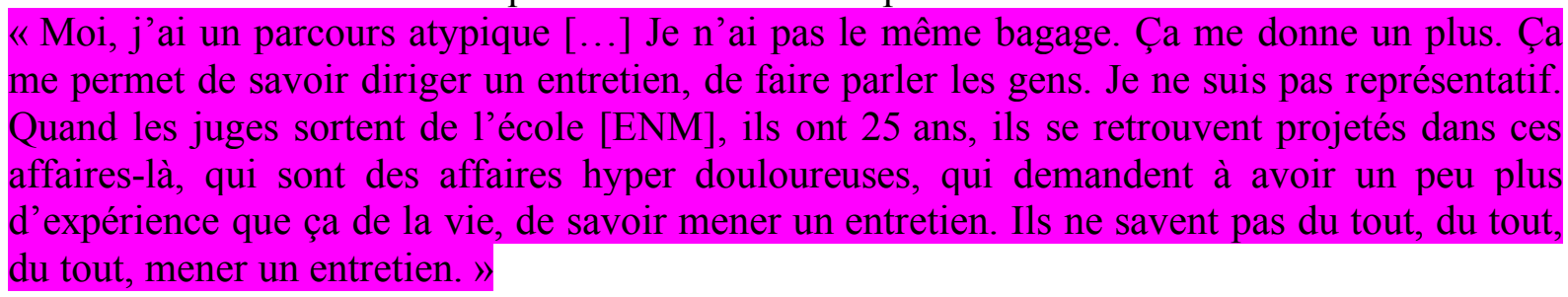

Y. Defert a une manière singulière d'exercer sa fonction, en assimilant parfois la position de juge à celle de psychologue et en assumant une conception quasi thérapeutique de son rôle. Il insiste sur la nécessité de prendre du temps (pour que les justiciables puissent « poser leurs valises »), sur l'importance du « tiers » dans la résolution des litiges familiaux. Contrairement à tous ses collègues, il pratique les auditions d'enfants en grand nombre, y compris avec des enfants en bas âge (dès deux ans et demi). Dans les propos qu'il tient en entretien, le vocabulaire psychologique a une grande place pour justifier ces auditions d'enfants : il évoque le « traumatisme » de la séparation, l'importance de la «parole transcendante » du juge, qui leur permet de " passer des caps », etc. Y. Defert considère que la masse des dossiers et la cadence à respecter dans le traitement des affaires gênent son travail. Comme d'autres collègues femmes, il met en œuvre des tactiques pour ralentir le rythme des procédures. Les auditions d'enfants et les enquêtes sociales lui permettent, par exemple, de revoir les justiciables une seconde fois et de suivre les affaires davantage dans la durée. Le juge Y. Defert est partisan des enquêtes sociales qui, selon lui, permettent aux justiciables de se « poser» en parlant à un psychologue. Il en ordonne en grand nombre (près de 130 par an) dès qu'il ne comprend pas une situation, nous dit-il, même s'il ne suit pas toujours les recommandations de l'expert. Comme les femmes JAF adoptant une posture pédagogique et interventionniste, Y. Defert insiste aussi sur la « circulation de la parole » dans la procédure : il met l'accent sur l'importance du moment de l'audience pour « dénouer les conflits » et se méfie des avocats qui, selon lui, «formatent» les discours des individus.

La conception du métier développée par le juge Y. Defert reprend en l'accentuant la posture pédagogique et interventionniste des femmes JAF. Cet exemple atypique permet ainsi de confirmer que ce n'est pas parce que le juge est un homme qu'il a forcément une absence d'intérêt pour le poste de JAF et des pratiques d'audiences aseptisées ou inquisitrices. À l'inverse, ce n'est pas parce que le juge est une femme qu'elle a forcément une conception pédagogique et interventionniste de sa fonction. Il n'y a donc pas un effet du sexe du juge en soi sur leurs représentations et leurs pratiques de leur métier. Cependant, les hommes et les femmes juges n'arrivent pas aux Affaires familiales dans les mêmes conditions, ni avec les mêmes expériences professionnelles, ni avec les mêmes projets de carrière, ni avec les mêmes contraintes (en particulier familiales). De ce fait, ils n'investissent pas cette fonction de la même façon. Pour le dire autrement, ce n'est pas du fait d'une «nature féminine » que les femmes juges s'intéressent aux aspects pratiques de la vie des justiciables et ce n'est pas du fait d'une « nature masculine » que les hommes juges défendent une vision surplombante de 
la fonction de magistrat. C'est parce que les juges femmes ont plus de probabilité que les juges hommes d'avoir (eu) à assumer des charges familiales dans leur vie personnelle, qu'elles sont plus sensibles aux problèmes pratiques des justiciables mis en avant aux Affaires familiales. C'est également parce que les juges femmes et hommes ne sont pas devenus magistrats pour les mêmes raisons, avec les mêmes objectifs (notamment en termes de vœux de fonction) que les premières ont plus de chance d'apprécier le poste de JAF que les seconds. Dans les manières différenciées d'endosser leur rôle par les juges se retrouve une pluralité des modes d'exercice des carrières selon le genre (Cacouault-Bitaud, 2001). Dans quelle mesure ces différences affectent-elles les décisions prises par les juges hommes et femmes aux affaires familiales?

\section{Féminisation de la magistrature et décisions aux Affaires familiales}

On touche ici une question sensible qui préoccupe les justiciables - qui craignent de tomber sur « le mauvais » ou « la mauvaise » juge pour leur affaire - mais aussi les professionnels de la Justice. La catégorie du sexe du juge paraît ainsi centrale dans la pratique des avocats majoritairement des avocates - qui revendiquent leur connaissance des juges et de leurs particularités comme élément de leur compétence, disant adapter leur plaidoirie au juge. « En deux ou trois audiences, on connait les manies des juges » nous explique ainsi Maitre Catherine Tortille, une avocate au barreau de Marjac : « $\mathrm{M}^{\text {me }}$ Placido est généreuse en pension alimentaire », " les deux [juges] hommes sont moins généreux », " une ancienne JAF ne supportait pas que les hommes payent davantage pour leur crédit auto que pour leur pension alimentaire ${ }^{48}$. Les catégories des avocates pour décrire les JAF — juges " pro-pères », " pro-mères », adeptes ou non de résidence alternée, fixant des pensions alimentaires plus ou moins élevées, etc. - reposent souvent sur le sexe du/de la JAF. La féminisation de la magistrature étant constituée en sujet de mobilisation de la part des associations de pères, certains tribunaux ont mis en place une politique explicite de masculinisation des postes de JAF.

\subsection{Des juges hommes nommés aux Affaires familiales}

C'est le cas au tribunal de Carly, un grand tribunal de la région parisienne, qui compte en 2009 neuf juges aux Affaires familiales, dont trois hommes. «Avec $\mathrm{M}^{\mathrm{me}} \mathrm{C}$. Blanchard [la présidente de la Chambre de la famille], on veut plus d'hommes aux Affaires familiales » nous explique d'emblée le président du TGI. Pour le justiciable, c'est important de ne pas toujours voir des femmes, cela évite certaines contestations judiciaires, par exemple celles menées par SOS-Papa. On essaye donc d'équilibrer $»^{49}$. Le juge E. Fratta est le dernier JAF arrivé à Carly, en septembre 2008. Sorti de l'ENM à 28 ans, il obtient la fonction de juge du siège en région parisienne qu'il souhaitait et raconte comment son affectation aux Affaires familiales est le résultat d'une politique volontariste de masculinisation de la part de la présidence du tribunal :

«Je pense qu'il y avait une volonté qu'il y ait un nouvel homme aux Affaires familiales,
parce que c'est une fonction très féminisée. Je pense que vous l'avez vu ou vous allez le voir,
ce n'est pas propre à Carly. Par exemple, il y avait une réunion de la Cour d'appel de Paris en
décembre dernier, et sur une quarantaine de JAF, nous étions 4 hommes et 36 femmes, et

\footnotetext{
${ }^{48}$ Informations recueillies au cours d'un entretien avec Maître C. Tortille à Marjac, réalisé par C. Bessière et S. N. Mohajer au cabinet, le 19 février 2009.

${ }^{49}$ Informations recueillies lors d'un entretien non-enregistré avec le président du tribunal de Carly, en présence de la secrétaire générale du TGI, réalisé par H. Steinmetz et J. Mandin, le 8 décembre 2008.
} 
parmi les 4 hommes il y en avait 3 de Carly ${ }^{50}$. »

Dans les quatre tribunaux où s'est déroulée l'enquête, le tribunal de Carly est le seul où nous avons observé une telle politique de masculinisation des Affaires familiales. Ce souci de mettre en avant la présence de juges hommes relève d'une volonté d'affichage destinée à soigner l'image des Affaires familiales vis-à-vis des justiciables. Quand on demande au juge E. Fratta ce qu'il pense de cette démarche volontariste, il dit que « cela répond à un ressenti dans la tête de pas mal de justiciables. [...] Il y a une proverbe anglais : la justice ne doit pas seulement être rendue, elle doit avoir l'apparence d'être rendue aussi ». Si à Carly, les JAF et leur hiérarchie se posent la question de l'influence du sexe du juge sur les décisions prises aux Affaires familiales, c'est pour y répondre par la négative. La politique mise en place se fonde davantage sur une inquiétude quant la visibilité des ces juges femmes (qui a longtemps conduit à privilégier pour les femmes magistrates des postes moins exposés au public [Boigeol, 1993]) que sur la conviction que les décisions rendues seraient différentes selon le sexe.

\subsection{Des décisions standardisées, quel que soit le sexe du juge}

De façon générale, dans les quatre tribunaux enquêtés, magistrats et magistrates sont prompts à rejeter toute influence du genre du juge sur les décisions prises aux Affaires familiales ${ }^{51}$. E. Fratta explique ainsi : «Globalement je ne dirais pas qu'il y a les pro-pères et les pro-mères [...] On est lié par les demandes. Vous savez, ce n'est pas parce que je suis un homme que ça change le fait que dans $70 \%$ des cas la résidence habituelle c'est chez la mère, parce que c'est la demande, le père est d'accord, c'est quand même $70 \%$ des cas $»$.

Notre enquête confirme ce point : les décisions rendues dans les Chambres de la famille des TGI sont d'une grande homogénéité, et ce quelque soit le sexe du magistrat. Dans la plupart des cas, lorsqu'il y a des enfants, la résidence habituelle est fixée chez la mère et s'accompagne du paiement d'une pension alimentaire du père vers la mère. Parmi les 276 dossiers concernant des enfants dans notre base de données, dans $73 \%$ des cas leur résidence habituelle est fixée chez la mère, dans $10 \%$ des cas chez le père, dans $13 \%$ c'est une résidence alternée et dans $4 \%$ la résidence des différents enfants n'est pas fixée chez le même parent. Cette répartition ne varie que très peu selon le sexe du juge (Figure 1). Le graphique présente la structure des 225 décisions prises par 31 magistrates et 51 décisions prises par 8 magistrats, tirées de façon aléatoire dans les quatre tribunaux enquêtés et concernant la résidence des enfants. Or, la structure des décisions - fixation massive de la résidence chez la mère, et de façon minoritaire fixation d'une résidence alternée ou chez le père - est identique dans les deux cas, avec $72 \%$ des décisions pour les magistrates femmes, et plus de trois quarts des décisions pour les magistrats hommes fixant la résidence chez la mère. La distribution est similaire pour les autres types de demande.

\section{Figure 1. Fixation de la résidence des enfants, selon le sexe du juge (en effectifs)}

Note : $\mathrm{N}=276$ décisions concernant la résidence des enfants, dont 225 ont été prises par seulement 31 magistrates ; et dont les 51 restantes ont été prises par 8 magistrats.

\footnotetext{
${ }^{50}$ Entretien avec le Juge E. Fratta, réalisé par H. Steinmetz et J. Mandin, le 27 janvier 2009.

${ }^{51}$ Seuls deux juges hommes - E. Paletot et P. Terreau, tous deux JAF au tribunal de Marjac — assument une certaine empathie et familiarité avec les justiciables hommes, allant même jusqu'à revendiquer un " point de vue masculin » dans leur fonction. Cette attitude que nous n'avons rencontrée chez aucun autre JAF homme ou femme doit sans doute être mise en regard avec le contexte de l'enquête ethnographique au tribunal de Marjac. Dans ce petit tribunal de province, au moment de l'enquête, ces deux hommes juges viennent juste d'être nommés aux Affaires familiales contre leur gré, en remplacement de quatre femmes JAF. Remarquons à la suite d'A. Boigeol (2010), qu'une telle posture serait impensable pour des femmes juges qui doivent bien plus souvent que les hommes faire face à l'accusation d'être partiales, du fait de leur sexe.
} 
Source : base des 400 dossiers archivés dans les 4 tribunaux de l'enquête (100 par tribunal) dans la période du 15 mai-15 juin et 15 octobre-15 novembre 2007.

Cette homogénéité des décisions s'explique d'abord par le cadre juridique qui en matière civile impose aux JAF de trancher dans un espace de possibilité ouvert par les demandes des parties et non au-delà. Or, les demandes justiciables, hommes et femmes sont plutôt uniformes, et se rejoignent pour demander majoritairement la fixation de la résidence des enfants chez la mère (62\% des demandes des pères, et $76 \%$ des demandes des mères) (Figure 2 ). Dans les audiences que nous avons observées, plus de 8 fois sur 10, le lieu de résidence des enfants n'est pas discuté du tout (et n'est donc pas un objet de contestation). Le ou la juge se contente d'entériner l'accord entre les parents, c'est-à-dire le plus souvent la résidence chez la mère et un «DVH classique » pour le père (soit un week-end sur deux et la moitié des vacances scolaires). À ce stade — à partir de notre base de données de 400 dossiers l'analyse statistique ne peut aller plus loin que le constat de cette similarité des structures des décisions de résidence des JAF hommes et femmes ${ }^{52}$. Cela permet cependant de constater que si les décisions des magistrats sont globalement similaires, c'est en partie pace qu'ils sont tenus par les demandes des justiciables.

Figure 2. Demandes des justiciables hommes et femmes en matière de résidence des enfants (en pourcentages)

Note : $\mathrm{N}=276$ dossiers concernant la résidence des enfants

Source : base des 400 dossiers archivés dans les 4 tribunaux de l'enquête (100 par tribunal) dans les périodes allant du 15 mai au 15 juin 2007 et du 15 octobre au 15 novembre 2007.

Les magistrates et les magistrats ont aussi des décisions similaires en termes de fixation de pensions alimentaire : dans notre base de données, les valeurs modales sont de $100 €$ à $150 €$ par mois et par enfant, quel que soit le sexe du juge. Les études économétriques disponibles confirment le très faible impact du sexe du juge sur ce point: les magistrates attribueraient, toutes choses égales par ailleurs, des pensions alimentaires supérieures en moyenne de $5 € /$ mois par rapport aux magistrats — cet effet étant de moindre importance que celui des revenus du parent contributeur ou celui des caractéristiques des enfants (Bourreau-Dubois et al., 2003, p. 61) ${ }^{53}$.

Confrontés à un contentieux massif et en augmentation, les JAF sont tenus par des objectifs de productivité qui portent sur le nombre de décisions à rendre, les délais de jugement, la réduction du stock de dossiers dans leur cabinet. Ils et elles développent deux types de

\footnotetext{
52 Pour aller plus loin, et notamment pour discuter des décisions des juges hommes et femmes, lorsque les demandes des parents en matière de résidence sont contrastées, il faudrait un échantillon de dossiers bien plus grand. En effet, parmi les 400 dossiers saisis, 276 décisions fixent la résidence des enfants, mais cela ne correspond pas forcément à des cas de désaccords entre les parents sur ce point. Dans 103 cas sur 137 affaires opposant les deux parents sur un aspect de leur séparation (comme la pension alimentaire, les droits de visite et d'hébergement, l'autorité parentale et enfin la résidence), soit pas moins des trois quart des cas, les parents ne formulent pas de demande différente en termes de résidence des enfants ; et parmi ces 103 cas, 89 concernent un accord sur la résidence chez la mère. Dans quatre dossiers, le père et la mère demandent chacun la résidence des enfants ; dans quatre dossiers le père demande une résidence alternée tandis que la mère demande une résidence exclusive. Contrairement aux représentations médiatiques, en particulier celles véhiculées par les associations de pères, une minorité des dossiers oppose le père et la mère à propos de la résidence des enfants.

${ }^{53}$ Ces études montrent que plus l'enfant est âgé, plus la pension alimentaire est importante. Par ailleurs, les pensions attribuées pour des filles sont en moyenne inférieures de $19 € /$ mois à celles attribuées pour des enfants de sexe masculin. Ce différentiel est congruent avec d'autres enquêtes qui montrent que les filles reçoivent un peu moins d'argent de poche que les garçons (Barnet-Verzat et Wolff, 2001), ainsi que des cadeaux moins coûteux (Vincent, 2001). En ce qui concerne les pensions alimentaires, l'effet du sexe de l'enfant est donc supérieur à celui du sexe du juge.
} 
pratiques de gestion de la masse : la standardisation des décisions courantes ${ }^{54}$ et la soumission à l'accord des justiciables ${ }^{55}$ pour pouvoir se consacrer aux quelques affaires " difficiles » qui exigent un traitement au cas par cas. Ces facteurs contribuent à une forte homogénéité des décisions des JAF, quel que soit leur sexe.

\section{Conclusion}

Pour conclure, l'analyse montre qu'il ne suffit pas pour comprendre l'influence du genre sur la production du droit par les juges de s'arrêter aux seules décisions prises. En effet, l'étude des décisions selon le genre ne permet pas de conclure à un effet clair du sexe du magistrat sur les jugements. En revanche, l'observation des pratiques des juges et l'analyse de leur parcours montrent l'existence de différences selon le genre dans la conception et l'exercice de leur rôle. D'un point de vue quantitatif, le cadre juridique et les contraintes organisationnelles qui s'imposent aux JAF les conduisent à prendre des décisions standardisées, quel que soit leur sexe. Notre recherche fait cependant émerger deux modèles genrés d'occupation de la fonction de JAF. Dans le modèle masculin - qui concerne plus souvent des hommes JAF mais aussi quelques femmes - les contraintes organisationnelles de productivité sont intériorisées par les juges. Il faut dire que les hommes JAF sont plus souvent dans des positions hiérarchiques élevées et responsables de l'application de ces critères quantitatifs d'évaluation du travail judiciaire. Le style aseptisé et procédural typique du modèle masculin - qui valorise l'accord entre les justiciables et met à distance leur intimité — permet de gagner du temps, mais ne garantit pas une grande satisfaction au travail. Dans le modèle masculin, les juges trouvent le contentieux des séparations répétitif et cherchent à quitter au plus vite les Affaires familiales pour faire carrière ailleurs. Le modèle féminin — porté surtout par des femmes JAF mais pas seulement - apparaît au contraire en porte-à-faux avec les objectifs de productivité imposés aux Chambres de la famille des TGI. Dans ce second modèle, les JAF utilisent les interstices de la procédure (renvois, enquêtes sociales, auditions d'enfants) pour suivre les dossiers sur le long terme. Le style pédagogique et interventionniste est coûteux en temps mais il permet de ré-enchanter la fonction, en donnant le sentiment aux JAF d'avoir une certaine utilité sociale. Au final, les décisions des uns et des autres sont proches, mais le processus judiciaire peut s'avérer finalement assez différent pour les justiciables, selon le style du juge à l'audience et dans ses jugements écrits — plus ou moins pédagogique, interventionniste, moralisateur, provocateur ou distant. Cela permet de voir que l'influence du juge ne se réduit pas seulement comme veulent l'imposer les associations de pères à une division entre juges pro-pères ou pro-pères selon le sexe. Au contraire, dans leur exercice de la fonction de juge en pratique, et dans ses effets sur l'expérience des justiciables, ce sont bien plutôt les manières de répondre aux contraintes institutionnelles de gestion de la masse qui différencient les juges selon leur genre. Le choix était alors de ne pas se centrer uniquement sur les décisions pour comprendre dans sa complexité le rapport des ces magistrats à l'exercice de leur profession. Ce n'est donc pas en postulant un effet mécanique de l'appartenance de sexe des juges que l'on peut comprendre les décisions prises en matière

\footnotetext{
${ }^{54}$ Dans le souci de réduire les différentiels entre juridictions en ce qui concerne la contribution à l'entretien des enfants, une grille de référence a été expérimentée à la Cour d'appel de Toulouse en 2009, puis diffusée sur l'ensemble du territoire en 2010. Cette grille est fondée à la fois sur l'évaluation du coût de l'enfant en fonction de l'âge, et sur les revenus du parent débiteur et du temps de résidence passé chez l'un et l'autre des parents. Elle a été construite à partir de l'étude citée plus haut (Bourreau-Dubois et al., 2003).

55 Cette «soumission" n'est pas complètement inscrite dans le droit puisque, même en cas de divorce par consentement mutuel, le/la juge doit vérifier que la convention proposée par les deux ex-époux est conforme aux règles de droit. Néanmoins, l'entérinement de l'accord permet aux juges de gagner du temps tout en validant un compromis qui leur semble d'autant plus viable puisqu'il a été proposé par les ex-conjoints eux-mêmes.
} 
familiale, mais en s'intéressant au processus judiciaire de séparation conjugale dans son ensemble et à sa manière de saisir les rapports de genre et les inégalités entre justiciables.

\section{Remerciements}

Cet article est issu d'une recherche collective sur le traitement judiciaire des séparations conjugales, qui a reçu le soutien de la Mission de recherche Droit et Justice ainsi que de l'ANR-12-JSH1-003-01-RUPTURES. Le texte a bénéficié des relectures et apports de nombreux collègues, notamment de Sophie Bernard, Émilie Biland, Anne Boigeol, Sibylle Gollac et Julie Minoc.

\section{Annexes}

La base de données a été constituée par le dépouillement et le codage de 400 dossiers tirés aléatoirement des archives des quatre tribunaux enquêtes, sur deux périodes de temps. Pour chaque dossier, un ensemble de plus de 300 variables a été codé, en détaillant dans chaque cas le sexe du magistrat, les décisions prises mais aussi les demandes des parties à partir des requêtes présentées dans les dossiers (et parfois complétées des plaidoiries des avocats). $C$ 'est ainsi qu'il nous a été possible de comparer les demandes réalisées par les hommes et par les femmes, ainsi que les décisions prises par les juges hommes et femmes. Dans de nombreux cas, la résidence n'est pas un objet de contestation, les conjoints demandent la même chose. Nous avons codé les demandes de des deux parents qui cö̈ncident alors sur ce point (mais la pension alimentaire, le droit de visite et d'hébergement ou l'autorité parentale peuvent être les enjeux du désaccord).

Tableau 1. Décisions des magistrats concernant la résidence selon le sexe, en pourcentages (et en effectifs)

\begin{tabular}{|l|c|c|c|c|c|}
\hline & $\begin{array}{c}\text { Résidence } \\
\text { alternée }\end{array}$ & $\begin{array}{c}\text { Résidence habituelle } \\
\text { chez la femme }\end{array}$ & $\begin{array}{c}\text { Résidence habituelle } \\
\text { chez l'homme }\end{array}$ & $\begin{array}{c}\text { Fratrie } \\
\text { séparée }\end{array}$ & Total \\
\hline Magistrates femmes & $12,44(28)$ & $72(162)$ & $11,11(25)$ & $4,44(10)$ & $100(225)$ \\
\hline Magistrats hommes & $15,69(8)$ & $76,47(39)$ & $5,88(3)$ & $1,96(1)$ & $100(51)$ \\
\hline Total & $\mathbf{1 3 , 0 4 ( 3 6 )}$ & $\mathbf{7 2 , 8 3 ( 2 0 1 )}$ & $\mathbf{1 0 , 1 4 ( 2 8 )}$ & $\mathbf{3 , 9 9 ( 1 1 )}$ & $\mathbf{1 0 0}(276)$ \\
\hline
\end{tabular}

\section{Tableau 2. Demandes des hommes et des femmes, en pourcentages (et en effectifs)}

\begin{tabular}{|l|c|c|}
\hline & Demandes des hommes & Demandes des femmes \\
\hline Résidence alternée & $19,25(41)$ & $12,65(31)$ \\
\hline Résidence habituelle chez la mère & $62,44(133)$ & $76,33(187)$ \\
\hline Résidence habituelle chez le père & $14,08(30)$ & $7,76(19)$ \\
\hline Fratrie séparée & $4,23(9)$ & $3,27(8)$ \\
\hline Total & $\mathbf{1 0 0}(213)$ & $\mathbf{1 0 0}(243)$ \\
\hline
\end{tabular}

Note : Dans un certain nombre de cas, plus nombreux pour les hommes que pour les femmes, aucune demande n'avait été renseignée. Certes, ces non-réponses peuvent être dues à une défaillance des chercheurs dans leur remplissage de la base de données. Mais elles peuvent signifier plus simplement l'absence de demande d'une partie en matière de résidence - ce qui équivaut dans un contentieux civil à un acquiescement tacite à la position de l'autre partie.

\section{Références}

Barnet-Verzat, C. et Wolff F.-C., 2001. L'argent de poche versé aux jeunes : l'apprentissage de l'autonomie financière. Economie et statistiques, 343, 3, pp. 51-72.

Bastard, B. 2010. Family mediation in France: a new profession has been established, but 
where are the clients? Journal of Social Welfare and Family Law, 32(2), pp. 135-142.

Bastard, B. Mouhanna C., 2007. Une justice dans l'urgence. Le traitement en temps réel des affaires pénales. PUF, Paris.

Benec'h-Le Roux, P., 2008. Au tribunal pour enfants. L'avocat, le juge, le procureur et l'éducateur. Presses universitaires de Rennes, Rennes.

Bessière, C., Gollac, S., Audot, R., Biland-Curinier, E., Coquard, B., Lignier, W., Louis, S., Mille, M., Minoc, J., Neuberg, S., Nouri-Mangold, S., Steinmetz, H. 2010. Au tribunal des couples. Les situations professionnelles d'hommes et de femmes au prisme des procédures judiciaires. Rapport final pour la Mission de recherche « Droit et Justice ». Ministère de la Justice, Paris.

Boigeol, A., 1993. La magistrature française au féminin : entre spécificité et banalisation. Droit et Société, 25, pp. 489-522.

Boigeol, A., 1996. Les femmes et les Cours. La difficile mise en œuvre de l'égalité des sexes dans l'accès à la magistrature. Genèses, 22, pp. 107-129.

Boigeol, A., 1997, Les magistrates de l'ordre judiciaire : des femmes d'autorité. Les cahiers du Mage, 1, pp. 25-35.

Boigeol, A., 2010. L'exercice de la justice au prisme du genre : un non-objet ? In : Cadiet L., Chauvaud, F., Gauvard, C., Schmitt Pantel P., Tsikounas M. (Eds.). Figures de femmes criminelles. De l'Antiquité à nos jours. Publications de la Sorbonne, Paris, pp. 329-343.

Bossis, R., 2003. La Question de la professionnalisation du corps des greffiers. Thèse en sociologie de l'Université Saint Quentin en Yvelines.

Botelho Junqueira, E., 2003. Women in the Judiciary : a perspective from Brazil. In : Schultz, U. et Shaw, G. (Eds.), Women in the World's Legal Profession. Hart Publishing, OxfordPortland (Oregon), pp. 437-450.

Bourreau-Dubois, C., et al. 2003. Les obligations alimentaires vis-à-vis des enfants de parents divorcés : une analyse économique au service du droit. Rapport pour la Mission de recherche « Droit et Justice » du Ministère de la Justice et de la Mission Recherche (MiRE) du Ministère de l'Emploi et de la Solidarité.

Cacouault-Bitaud, M., 2001. La féminisation d'une profession est-elle le signe d'une baisse de prestige ? Travail, genre et sociétés, 5, pp. 91-115.

Champy, F., 2009. La Sociologie des professions. PUF, Paris.

Christin, A., 2008. Comparutions immédiates. Enquête sur une pratique judiciaire. La Découverte, Paris.

Clot, Y., 2010. Le Travail à cœur: pour en finir avec les risque psychosociaux. La Découverte, Paris.

Dekeuwer-Defossez, F., 1999. Rénover le droit de la famille. Propositions pour un droit adapté aux réalités et aux aspirations de notre temps. Rapport au Garde des sceaux, ministre de la Justice. La Documentation française, Paris.

Feenan, D., 2009. Women and Judging. Feminist Legal Studies, 17, p. 1-9.

Hochschild, A., 1983. The Managed Heart: Commercialization of Human Feeling. University of California Press, Berkeley.

Hunter, R., 2013 (à paraître). Justice Marcia Neave : Case Study of a Feminist Judge. In Schultz U. et G. Shaw G. (Eds.), Gender and Judging. Hart Publishing, Oxford-Portland (Oregon).

Kohen, B., 2008. Family judges in the city of Buenos Aires: a View From Within. International Journal for the Legal Professions, 15(1-2), pp. 111-122.

Laufer J., 2004. Femmes et carrières : la question du plafond de verre. Revue française de gestion, 4(151), pp. 117-127.

Lelièvre, M., Léonard, T., 2012. Une femme peut-elle être jugée violente ? Les représentations de genre et les conditions de leur subversion lors des procès en comparution 
immédiate. In : Cardi C. et Pruvost G. (Eds), Penser la violence des femmes. La Découverte, Paris, pp. 314-329.

Mary, F.L., 1996. Femmes, délinquance et contrôle pénal. Analyse socio-démographique des statistiques administratives françaises. CESDIP, Guyancourt, «Etudes et données pénales », 75.

Moreau, C., 2010. Les avocats : une profession en expansion qui se féminise. Infostat Justice, 109.

Pruvost, G., 2007. Profession : policier. Sexe : féminin. MSH, Paris.

Rennes, J., 2007. Le mérite et la nature. Une controverse républicaine : l'accès des femmes aux professions de prestige, 1880-1940. Fayard, Paris.

Testenoire, A., 2001. Les carrières féminines : contingence ou projet ? Travail, genre et sociétés, 5, pp. 117-133.

Théry, I., 1993. Le Démariage. Odile Jacob, Paris.

Vauchez A., 2008. Le chiffre dans le «gouvernement» de la Justice. Revue française d'administration publique, 125, pp. 111-120.

Vincent S., 2001. Le Jouet et ses usages sociaux. La Dispute, Paris.

Vigour C., 2011. Choix politiques et inertie des dépenses. L'augmentation et les efforts de maîtrise du budget de la justice (1980-2010). In : Bezes P. et Siné A. (Eds.), Gouverner (par) les finances publiques. Presses de Sciences Po, Paris.

Zolezio, E., 2009. Des femmes dans un métier d'hommes : l'apprentissage de la chirurgie. Travail, genre et sociétés, 22, pp. 117-133. 